\title{
Neural Analog of Arousal: Persistent Conditional Activation of a Feeding Modulator by Serotonergic Initiators of Locomotion
}

\author{
Jian Jing, Ferdinand S. Vilim, Elizabeth C. Cropper, and Klaudiusz R. Weiss \\ Department of Neuroscience, Mount Sinai School of Medicine, New York, New York 10029
}

\begin{abstract}
We investigated how a neural analog of a form of arousal induced by a mildly noxious stimulus can promote two antagonistic responses, locomotion and feeding. Two pairs of cerebral serotonergic interneurons in Aplysia, CC9 and CC10, were persistently activated by transient noxious stimuli. Direct stimulation of CC9-10 activated locomotor activity that outlasted the stimulation and enhanced subsequent nerve-evoked locomotor programs. Thus, CC9-10 function both as initiators and as modulators of the locomotor network. CC9-10 also interacted with the feeding circuit but in a fundamentally different manner. CC9-10 did not directly trigger feeding activity or activate feeding command or pattern generating interneurons. CC9-10 did, however, elicit slow EPSPs in serotonergic cells that modulate feeding responses, the metacerebral cells (MCCs). CC9-10 persistently enhanced MCC excitability, but did not activate the MCCs directly. Previous work has demonstrated that the MCCs are activated during food ingestion via a sensory neuron C2. Interestingly, we found that CC9-10 stimulation converted subthreshold C2 mediated excitation of the MCC into suprathreshold excitation. Transient noxious stimuli also enhanced MCC excitability, and this was largely mediated by CC9-10. To summarize, CC9-10 exert actions on the feeding network, but their functional effects appear to be conditional on the presence of food-related inputs to the MCCs. A potential advantage of this arrangement is that it may prevent conflicting responses from being directly evoked by noxious stimuli while also facilitating the ability of food-related stimuli to generate feeding responses in the aftermath of noxious stimulation.
\end{abstract}

Key words: arousal; feeding; locomotion; Aplysia; neuromodulation; excitability; serotonin

\section{Introduction}

At any given moment, the decision to generate a specific behavior is determined both by sensory input and the current internal state. Decisions based on immediate effects of stimuli (e.g., behavioral switching) are generally simple because they are largely implemented by inhibitory interactions between premotor and pattern generating elements of distinct networks (Kovac and Davis, 1977; Edwards, 1991; Norekian and Satterlie, 1996; Shaw and Kristan, 1997; Jing and Gillette, 2000; Jing and Weiss, 2001). These inhibitory interactions prevent a conflict between competing responses. In contrast, decisions that rely on persistent changes in network states (e.g., arousal) can be complicated by the fact that arousal can exist in multiple forms that are triggered by different types of stimuli and have different degrees of specificity (Weiss et al., 1982; Robbins and Everitt, 1995; Pfaff, 2006). For instance, arousal induced by food or a mate promotes activity in a limited number of networks. In contrast, arousal induced by a noxious stimulus (such as a shock) may increase the probability of evoking multiple, sometimes even antagonistic, classes of be-

Received Aug. 13, 2008; revised Sept. 29, 2008; accepted 0ct. 15, 2008

This work was supported by the National Institutes of Health Grants R01 MH 36730, DA 13330, and MH 51393. We thank Jacqueline Jing for technical assistance.

Correspondence should be addressed to Dr. Jian Jing, Department of Neuroscience, Box 1065, Mount Sinai School of Medicine, 1 Gustave Levy Place, New York, NY 10029. E-mail: jingj01@gmail.com. DOI:10.1523/JNEUROSCI.3855-08.2008

Copyright $\odot 2008$ Society for Neuroscience $\quad 0270-6474 / 08 / 2812349-13 \$ 15.00 / 0$ haviors. More specifically, moderately noxious or threatening stimuli can promote both defensive behaviors (e.g., locomotion) and competing behaviors such as feeding (Antelman and Szechtman, 1975; Kalén et al., 1989), grooming (Rowell, 1961), and sexual responses (Barfield and Sachs, 1968). To our knowledge, there is no clear identification of mechanisms that could allow a form of arousal to promote antagonistic behaviors yet prevent a conflict between them.

We examine this issue in Aplysia, where noxious stimuli such as tail pinch that elicits locomotion also promote feeding responses (Kupfermann and Weiss, 1981). In principle, if neurons implementing arousal of the locomotor network were to activate the command or pattern generating neurons for feeding, this could trigger feeding in the absence of food and as consummatory feeding responses inhibit locomotion (Kupfermann, 1974), this could interrupt locomotion prematurely. We hypothesized that interruption of locomotion could be avoided if the locomotor network up-modulated the excitability (cf. Zhang and Linden, 2003) of the neurons that mediate food induced arousal (rather than directly exciting the network that generates feeding). This would insure that feeding responses would be facilitated but only in the presence of food. Food induced-arousal in Aplysia is mediated by a pair of serotonergic metacerebral cells (MCCs) (Weiss et al., 1978; Rosen et al., 1989; Morgan et al., 2000; Proekt and Weiss, 2003). However, the identity of neurons that mediate the nociception-activated arousal for locomotion was unknown. 

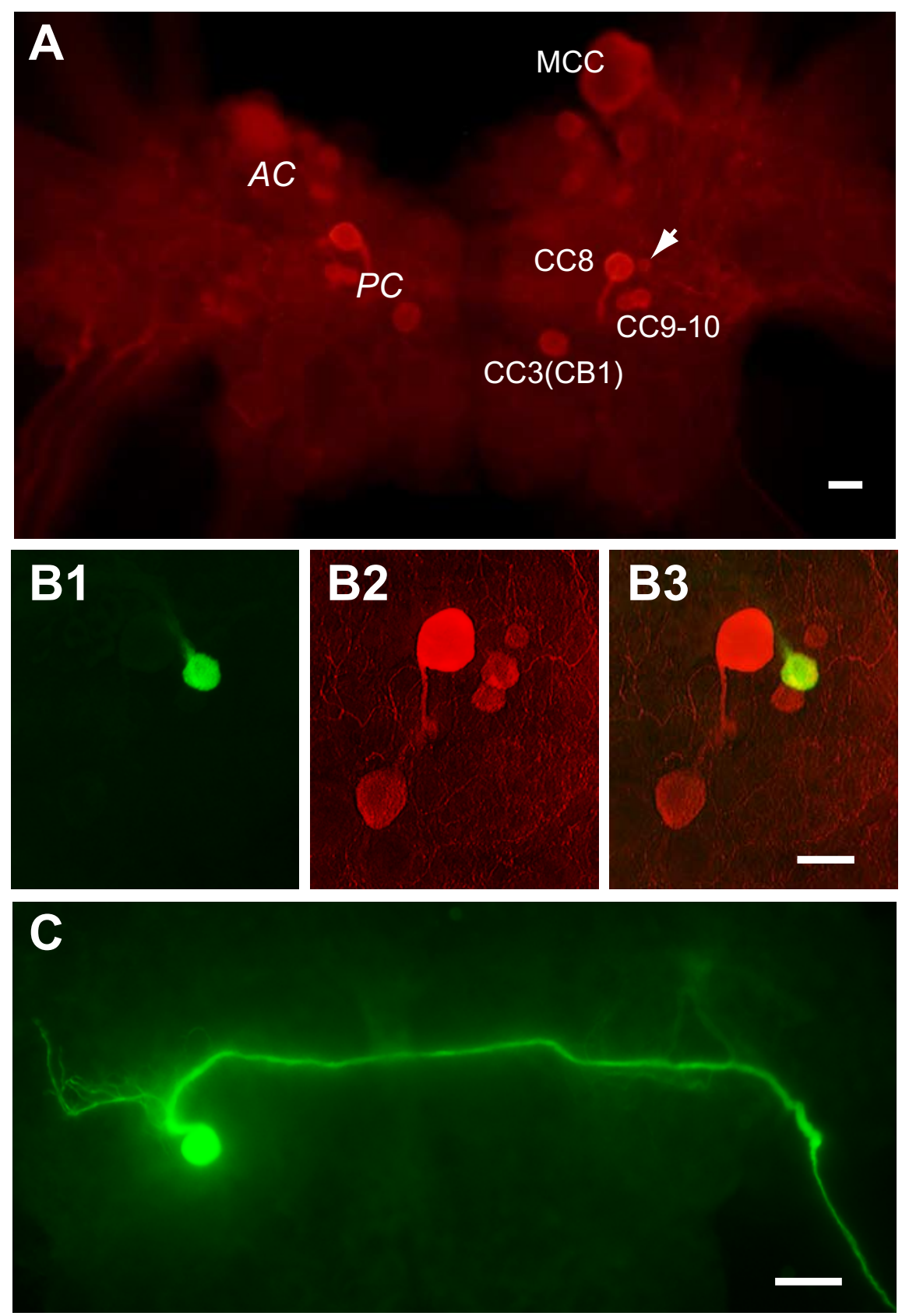

Figure 1. Serotonin immunoreactivity and morphology of $\mathrm{C}(9 / 10$. $A$, Distribution of serotonergic neurons in the cerebral ganglion demonstrated by serotonin immunocytochemistry (Red, Rhodamine Red X). There are two clusters of serotonergic cells: the anterior cluster (AC) and the posterior cluster (PC) (labeled at the left side of the cerebral ganglion). AC includes the MCC and smaller cells adjacent to it, the small cells are mostly located near the ventral surface and are out of focus. The PC includes five cells: $C \mathrm{C} 8, C \mathrm{C} 3(\mathrm{CB} 1), C \mathrm{C}$, CC10 and an unidentified cell (arrow). B, CC9/10 (green, carboxyfluorescein, $B 1$ ) is serotonin immunoreactive (Red, Rhodamine Red X) (B2). B3, Overlaid image of $\boldsymbol{B} 1$ and $\boldsymbol{B} 2$ showing the double labeled $C(C 9 / 10$ is yellowish. Right cerebral ganglion. $C$, Axon projection of a left $C(9 / 10$ revealed by carboxyfluorescein injection. $C(9 / 10$ processes ramify anteriorly in the vicinity of the soma. The main axon then turns medially to cross the cerebral commissure and exit the contralateral cerebro-pedal connective. Scale bars, $100 \mu \mathrm{m}$. All panels oriented with anterior at top.

We identify two pairs of cerebral serotonergic interneurons, CC9 and $\mathrm{CC} 10$, that are persistently activated by a variety of noxious stimuli. Within the locomotor circuit, CC9-10 function both as drivers and persistent modulators of the network, primarily by enhancing the ability of the network to be activated. Outside the locomotor network, they promote activity of the MCC through persistent increases in MCC excitability that result in a conditional increase in MCC firing. These simple mechanisms may allow the CNS to increase the probability of sequentially generating two responses without creating a conflict between them.

\section{Materials and Methods}

Aplysia californica were obtained from Marinus Scientific and maintained in circulating artificial seawater (ASW) at $14-15^{\circ} \mathrm{C}$.

Antibodies. We initially used two different serotonin antibodies, a commercial rabbit antibody (Immunostar) and a rat antibody that we generated. The staining patterns observed with these two antibodies were indistinguishable, and we primarily used the rat antibody. We generated the rat antibody using a modification of a procedure described previously (Furukawa et al., 2001; Sweedler et al., 2002). Briefly, the antigen was prepared by coupling $2 \mathrm{mg}$ serotonin oxalate to $10 \mathrm{mg}$ BSA (Sigma-Aldrich) in 1 $\mathrm{ml}$ of $50 \mathrm{~mm} \mathrm{NaH}_{2} \mathrm{PO}_{4}$, pH 7.2, using $100 \mu \mathrm{l}$ $16 \%$ paraformaldehyde (EMS). Following overnight incubation at $4^{\circ} \mathrm{C}$, coupled antigen was purified from the reaction using a Microcon-30 spinning at $13,800 \times \mathrm{g}$ for $30 \mathrm{~min}$ at $4^{\circ} \mathrm{C}$. After washing the retentate four times with $0.4 \mathrm{ml}$ of $50 \mathrm{~mm} \mathrm{NaH} \mathrm{PO}_{4}$, it was resuspended in $0.5 \mathrm{ml}$ of the same buffer and transferred to a new tube.

Two male Sprague Dawley rats $(250-300$ g; Taconic) were immunized by intraperitoneal injection with $12.5 \mu \mathrm{l}(250 \mu \mathrm{g})$ of antigen in an emulsion of $0.3 \mathrm{ml}$ of PBS and $0.3 \mathrm{ml}$ of Freund's complete adjuvant. At days 21 and 42, the rats were boosted by intraperitoneal injection with $6.25 \mu \mathrm{l}(125 \mu \mathrm{g})$ of antigen in the emulsion as above. The animals were killed by decapitation at $49 \mathrm{~d}$, and the blood was harvested and processed for serum. Sera were aliquoted, frozen and lyophilized, or stored at $4^{\circ} \mathrm{C}$ with EDTA ( $25 \mathrm{~mm}$ final concentration) and thimerosal ( $0.1 \%$ final concentration) added as stabilizers. Only one of the two rats produced a viable antibody for serotonin immunostaining. Preincubation of the antibody with $100 \mu \mathrm{g} / \mathrm{ml}$ of serotonin coupled to BSA (as above) abolished immunostaining, but adsorption with BSA coupled to glutamate instead of serotonin (as above) did not. This adsorption control, as well as the similarities between the staining pattern observed with our rat antibody and the staining pattern observed with the previously characterized commercial antibody indicates that our rat antibody is specific.

Immunocytochemistry. Immunocytochemistry was performed as described previously (Jing et al., 2007). Tissues were fixed in $4 \%$ paraformaldehyde, $0.2 \%$ picric acid, $25 \%$ sucrose, and $0.1 \mathrm{M} \mathrm{NaH}_{2} \mathrm{PO}_{4}, \mathrm{pH} 7.6$, for either $3 \mathrm{~h}$ at room temperature or overnight at $4^{\circ} \mathrm{C}$. All subsequent incubations were done at room temperature. The tissue was washed with PBS, and was permeabilized and blocked by overnight incubation in blocking buffer (BB; 10\% normal donkey serum, 2\% Triton X-100, 1\% BSA, $154 \mathrm{~mm}$ $\mathrm{NaCl}, 50 \mathrm{~mm}$ EDTA, $0.01 \%$ thimerosal, and $10 \mathrm{~mm} \mathrm{Na}_{2} \mathrm{HPO}_{4}, \mathrm{pH} 7.4$ ). The primary antibody was diluted 1:250 in $\mathrm{BB}$ and incubated with the tissue for $4-7 \mathrm{~d}$. The tissue was then washed twice per day for $2-3 \mathrm{~d}$ with washing buffer (WB; 2\% Triton X-100, 1\% BSA, $154 \mathrm{~mm} \mathrm{NaCl}, 50 \mathrm{~mm}$ EDTA, $0.01 \%$ thimerosal, and $10 \mathrm{~mm} \mathrm{Na}_{2} \mathrm{HPO}_{4}, \mathrm{pH}$ 7.4). After washing, the tissue was incubated with a 1:500 dilution of secondary antibody 
(lissamine rhodamine donkey anti-rat; Jackson ImmunoResearch) for 2-3 d and then washed again two times with WB for $1 \mathrm{~d}$ and four times with storage buffer (1\% BSA, $154 \mathrm{~mm} \mathrm{NaCl}, 50$ mM EDTA, $0.01 \%$ thimerosal, and $10 \mathrm{~mm}$ $\mathrm{Na}_{2} \mathrm{HPO}_{4}, \mathrm{pH}$ 7.4) for $1 \mathrm{~d}$.

For double labeling experiments, identified neurons were injected with carboxyfluorescein (Jing et al., 2003), and the tissue was fixed and processed for immunocytochemistry as described above.

Isolated CNS preparations. The cerebral ganglion and pleural-pedal ganglia were pinned in a sylgard dish with a volume of $\sim 1.5 \mathrm{ml}$. In all experiments, the dorsal surface of the cerebral ganglion was desheathed to expose the cells of interest. In experiments where connections from CC9-10 to pedal neurons were examined, the pedal ganglion was also desheathed. In experiments that tested connections from CC9/10 to cerebral-buccal interneurons (CBIs) the cerebral and buccal ganglia were retained. Both the dorsal and the ventral surfaces of the cerebral ganglion were desheathed. The cerebral ganglion was then twisted at the commissure to make it possible to access CC9/10 on the dorsal surface and contralateral CBIs on the ventral surface. Nerve recording and stimulation was done through suction electrodes made from polyethylene tubing. The preparation was continuously perfused with ASW (in mм: 460 $\mathrm{NaCl}, 10 \mathrm{KCl}, 55 \mathrm{MgCl}_{2}, 11 \mathrm{CaCl}_{2}$, and 10 HEPES buffer, $\mathrm{pH}=7.6$ ) at $0.3 \mathrm{ml} / \mathrm{min}$ and maintained at $14-17^{\circ} \mathrm{C}$.

Fictive locomotor activity was elicited by stimulating nerve $\mathrm{P} 9$, which innervates the tail, or branch 4 of the Anterior Tentacular Nerve $\left(\mathrm{AT}_{4}\right)$, which innervates the tentacle. The stimulation paradigm was as follows: $20 \mathrm{~Hz}$, of $3 \mathrm{~ms}, 10-15 \mathrm{~V}$ pulses for $3 \mathrm{~s}$. Locomotor activity was recorded from the parapedal commissure nerve (PPCN), which has been shown previously to be a reliable monitor of locomotor activity (Jahan-Parwar and Fredman, 1980; Fredman and Jahan-Parwar, 1983). Locomotor activity in the PPCN is represented as a series of bursts. To calculate cycle frequency, for a given number of bursts, "n"; we measured the time, "t", that elapsed from the first burst to the last burst, and cycle frequency $=(\mathrm{n}-1) / \mathrm{t}$. Program latency of the locomotor activity was calculated as the time that elapsed from the end of nerve stimulation to the onset of the first burst.

Assays of monosynaptic synaptic connections were conducted in highdivalent saline (in mu: $312 \mathrm{NaCl}, 10 \mathrm{KCl}, 132 \mathrm{MgCl}_{2}, 33 \mathrm{CaCl}_{2}$, and 10 HEPES, $\mathrm{pH}=7.6$ ), solutions that elevate spike threshold and thus reduce polysynaptic activation. Neurons were identified based on location, size, electrophysiological and morphological characteristics.

Semi-intact preparations. Animals were anesthetized by injections ( $25 \%$ of body weight) of isotonic $\mathrm{MgCl}_{2}$. An incision was made from the dorsal side. The gut was separated from the rest of the animal at the level of the esophagus. The head, including tentacles, rhinophores, and buccal mass, together with the CNS (the cerebral ganglion, buccal ganglion, and pleural-pedal ganglion) were cut from the rest of the animal at the level of the rhinophores (dorsally) and slightly caudal to the beginning of the foot (ventrally). The head structure remained innervated by the Upper Labial Nerve and AT, and the buccal mass remained innervated by all buccal nerves. The preparation was placed in a two chamber sylgard-lined dish with the head in the larger chamber and the CNS in the smaller chamber, which had a higher sylgard floor. A subset of preparations also included the tail, which was isolated and attached to the pedal ganglion through bilateral P9 nerves. The tail was placed in the same chamber as the head. The sheath over the dorsal surface of the cerebral ganglion was removed to gain access to CC9/10 and MCC.

The buccal artery was cannulated to allow continuous perfusion of the head and buccal mass with cooled fresh ASW with glucose (in mM: 460 $\mathrm{NaCl}, 10 \mathrm{KCl}, 55 \mathrm{MgCl}_{2}, 11 \mathrm{CaCl}_{2}, 5$ glucose, and 10 HEPES buffer, $\mathrm{pH}=$ 7.6) at $\sim 2 \mathrm{ml} / \mathrm{min}$ using a bubble separator in the perfusion line. The buccal mass pressure was monitored with a pressure transducer, which had a probe placed in the bubble separator. Buccal mass pressure has been shown to correspond well with feeding movements (Weiss et al., $1986 \mathrm{~b})$. The preparation was maintained at $14-16^{\circ} \mathrm{C}$. All chemicals were purchased from Sigma.

Feeding behavior was elicited by touching the mouth with cut pieces of dried seaweed held by forceps. Pieces of seaweed were $\sim 3.5 \times 24 \mathrm{~mm}$. A typical feeding sequence consisted of initial biting responses and late swallowing responses which were initiated if the seaweed was successfully grasped. Completion of swallowing was verified by visual observation of the seaweed moving out of the cut end of the esophagus. We used forceps to touch the tentacle, and also to pinch the tentacle and the tail.

Data analysis. Digitized electrophysiological recordings were plotted using Coreldraw and Axum (Mathsoft). Digital photographic images were processed using Abode Photoshop and Illustrator. Data are expressed as mean \pm SEM. Differences between two data groups were tested with two-tailed paired or unpaired $t$ test. Data with more than two groups were first analyzed with one way ANOVA. Dunnett corrections were used for individual post hoc comparisons between groups. All statistical tests were performed using GraphPad Prism 4.0.

\section{Results}

Serotonin promotes both locomotion and feeding in Aplysia (Palovcik et al., 1982; Mackey and Carew, 1983; Kabotyanski et al., 2000). In the feeding system, serotonin is expressed in the MCC neurons (Eisenstadt et al., 1973; Weinreich et al., 1973), which mediate food-induced arousal of the feeding network (Kupfermann and Weiss, 1982; Rosen et al., 1989). The MCCs do not, however, trigger locomotor activity or modulate the loco- 
A P9 stimulation

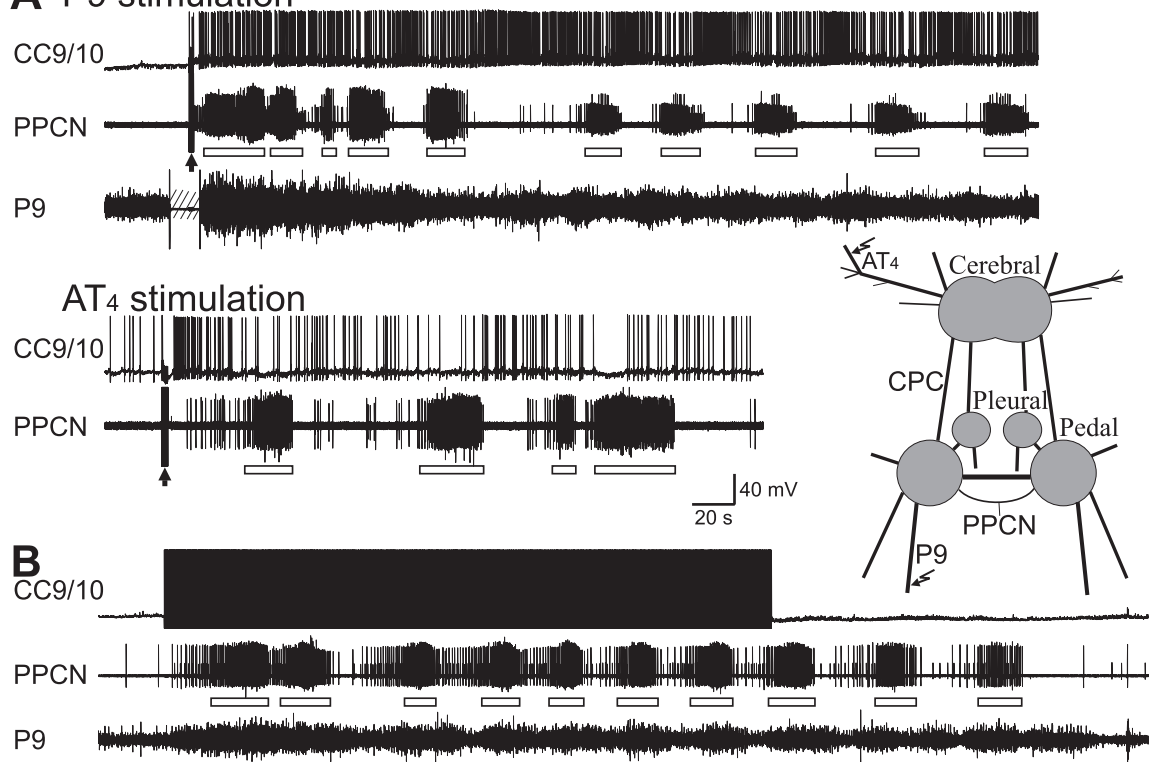

Figure 3. $C(\mathrm{C} 9 / 10$ is activated during nerve-evoked fictive locomotion and can induce fictive locomotion on its own. $A, C(9 / 10$ was activated for several minutes by a $3 \mathrm{~s}, 20 \mathrm{~Hz}$ stimulation of the tail nerve (P9) or branch 4 of AT (arrows). CC $9 / 10$ activity coincided with fictive locomotor activity (or pedal-wave motor program) as monitored by bursting activity (open bars) in PPCN. The line drawing at the right shows the major ganglia: the cerebral ganglion, the pleural-pedal ganglia, and the nerves that were stimulated ( $\mathrm{P9}$ and $\mathrm{AT}_{4}$ ) and used for recording (PPCN and P9). CPC, cerebro-pedal connective. B, Stimulation of $\mathrm{CC} 9 / 10$ at $5 \mathrm{~Hz}$ for 4.7 min elicited fictive locomotor activity in PPCN, which was similar to the activity elicited by nerve stimulation.

motor network. We therefore sought to identify other serotonergic neurons that could serve this purpose.

\section{Identification}

Both the cerebral and the pedal ganglia are involved in locomotion (Hening et al., 1979; Jahan-Parwar and Fredman, 1979, 1980; Fredman and Jahan-Parwar, 1980). Serotonergic neurons in the pedal ganglion modulate pedal muscle contractions (McPherson and Blankenship, 1992), but do not appear to initiate locomotion. We therefore focused on the cerebral ganglion.

A number of studies have used 5-HT immunocytochemistry to identify two major clusters of serotonergic neurons in the cerebral ganglion of Aplysia (Ono and McCaman, 1984; Longley and Longley, 1986; Nolen and Carew, 1994; Fickbohm et al., 2001), a pattern similar to several other gastropod mollusks (Panchin et al., 1995; Satterlie and Norekian, 1995; Sudlow et al., 1998; Jing and Gillette, 1999; Newcomb et al., 2006; Tian et al., 2006). Figure $1 A$ shows the distribution of serotonergic neurons on the dorsal surface of the cerebral ganglion (see also Fickbohm et al., 2001). In the anterior cluster (within the G cluster), there is a giant serotonergic neuron, the MCC, which sends its axon to the buccal ganglion and plays an important role in modulating the feeding network (Weiss et al., 1978, 1986b; Rosen et al., 1989). Adjacent to the MCC, there are several smaller serotonergic neurons that are often located closer to the ventral surface. The posterior cluster (within the C cluster), located near the commissure, contains 5 serotonergic neurons. Two of these have been described previously: CC3(CB1) (Mackey et al., 1989; Xin et al., 2001) and CC8 (Marinesco et al., 2004). This report focuses on two of the other three serotonergic neurons, which we have given the designations CC9 and CC10. These two neurons are adjacent cells that have been previously identified and described in an abstract (McPherson and Katz, 2001). Data shown in Figure $1 B$ confirm that CC9 and CC10 are serotonin immunoreactive $(n=8)$.

To facilitate physiological studies of CC9-10, we characterized additional features that distinguish these neurons from neighboring cells. CC9 and CC10 are medium sized pale neurons, often without pigmentation. Intracellular dye injections ( $n=4)$ indicate that they project their main axons to the contralateral pedal ganglion through the cerebro-pedal connective (Fig. 1C). Furthermore, CC9 and CC10 are electrically coupled, with an average steady-state coupling ratio of $0.099 \pm 0.015(n=5)$ (Fig. $2 A)$, and are electrically coupled to their contralateral homolog, with an average steady-state coupling ratio of $0.061 \pm 0.005(n=3)$. The two neurons are very similar to each other and cannot be distinguished. We refer to them singly as CC9/10 when we record from only one cell. Collectively we refer to the two cells in a single hemiganglion as CC9-10. CC9-10 often exhibit periodic spontaneous activity and fire at low rates $(<1.5 \mathrm{~Hz})$. Frequent IPSPs are observed simultaneously in both cells (Fig. $2 B$ ). Activation of one CC9/10 can induce polysynaptic inhibition in the other CC9/10 (Fig. 2C1). If the excitatory influence of the electrical coupling between the two cells is reduced by using short current pulses to activate spikes in a CC9/10 (instead of DC current injection), the polysynaptic inhibition is more apparent. Under these conditions, it hyperpolarizes not only the postsynaptic CC9/10, but also itself (Fig. 2C2).

\section{CC9-10 activity in response to noxious stimuli and initiation of locomotor activity}

Although CC9 and CC10 neurons are located in the cerebral ganglion, they project to the pedal ganglion where the central pattern generator (CPG) for locomotion is located (Hening et al., 1979; Jahan-Parwar and Fredman, 1979, 1980). We hypothesized therefore that CC9-10 play a role in initiating locomotion. To test this possibility we initially characterized responses of CC9 and CC10 to noxious stimuli that effectively evoke locomotor responses.

In a first series of experiments, we elicited fictive locomotor activity, or pedal wave motor programs, by stimulating two peripheral nerves that innervate the tail and the head (the tail nerve and anterior tentacular nerve, respectively) and observed activity of CC9/10 during locomotion. The tail nerve (P9) of the pedal ganglion conveys sensory information from the tail. Tail shock or direct stimulation of P9 mimics noxious sensory inputs and has been used extensively as a reinforcement for sensitization paradigms (Walters et al., 1983; Mackey et al., 1989; Marinesco et al., 2004). Branch 4 of the AT $\left(\mathrm{AT}_{4}\right)$ carries sensory information from the head (Lechner et al., 2000), and stimulation of this nerve has been used as a conditioned stimulus for classic conditioning of feeding responses (Lechner et al., 2000; Mozzachiodi et al., 2003; Brembs et al., 2004).

In isolated CNS preparations, we stimulated $\mathrm{P} 9$ at $20 \mathrm{~Hz}$ for $3 \mathrm{~s}$ to initiate locomotor activity which was monitored by cyclic bursting activity in the para-pedal commissural nerve (PPCN) 
A
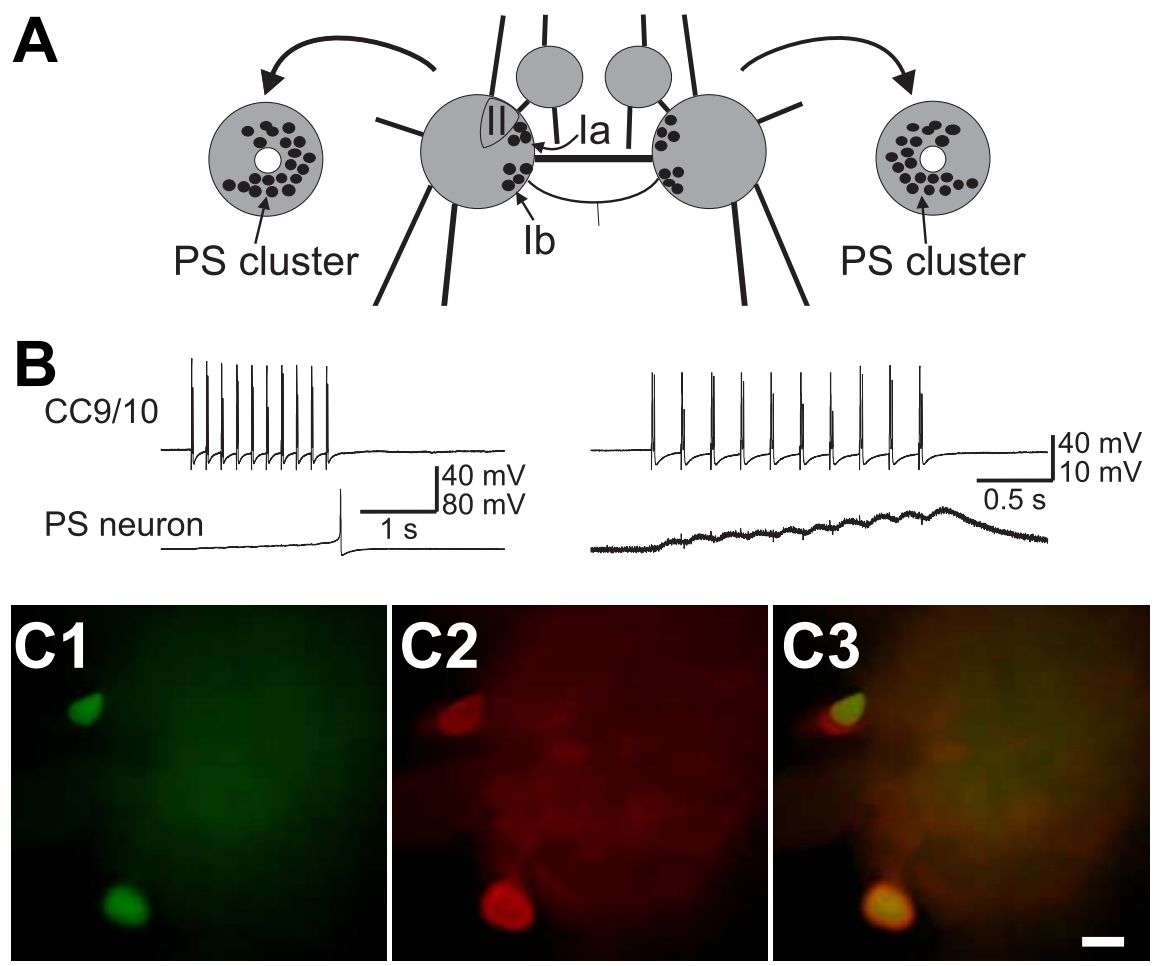

Figure 4. $\quad C(9 / 10$ excites serotonergic modulatory neurons in the pedal ganglion. $\boldsymbol{A}$, Schematic diagram of the pleural-pedal ganglia (dorsal view) illustrating the approximate locations of the pedal cells recorded from $\boldsymbol{B}$ : sector la, Ib and II. Drawings at the left and right are the bilateral pedal ganglia with the pedal commissure (PC) cut, and turned $90^{\circ}$ laterally so that the PC faces out (shown as the white circles at the center) to reveal the PS cluster neurons located on the ventral surface of the pedal ganglion (modified from Marinesco et al., 2004). B, Stimulation of (C9/10 elicited a spike in a pedal serotonergic (PS) neuron (left). When the PS neuron was hyperpolarized by $10 \mathrm{mV}$ from its resting potential to prevent it from spiking, fast EPSPs that followed presynaptic spikes one-for-one were observed (right). Recordings were made in high divalent saline. C, Two PS neurons on the dorsal surface of a right pedal ganglion that received fast EPSPs from $C(9 / 10$ were injected with carboxyfluorescein dye (C1), and were serotonin immunoreactive (C2). C3, Overlaid image of $\mathbf{C}$ and $\mathbf{C}$ showing the double labeled PS neurons are yellowish. A third PS neuron located below the top cell was also serotonin immunoreactive. The top two cells are located in the sector la, while the lower cell is located in the sector lb. Scale bar, $200 \mu \mathrm{m}$. Anterior at top, pedal commissure facing left.

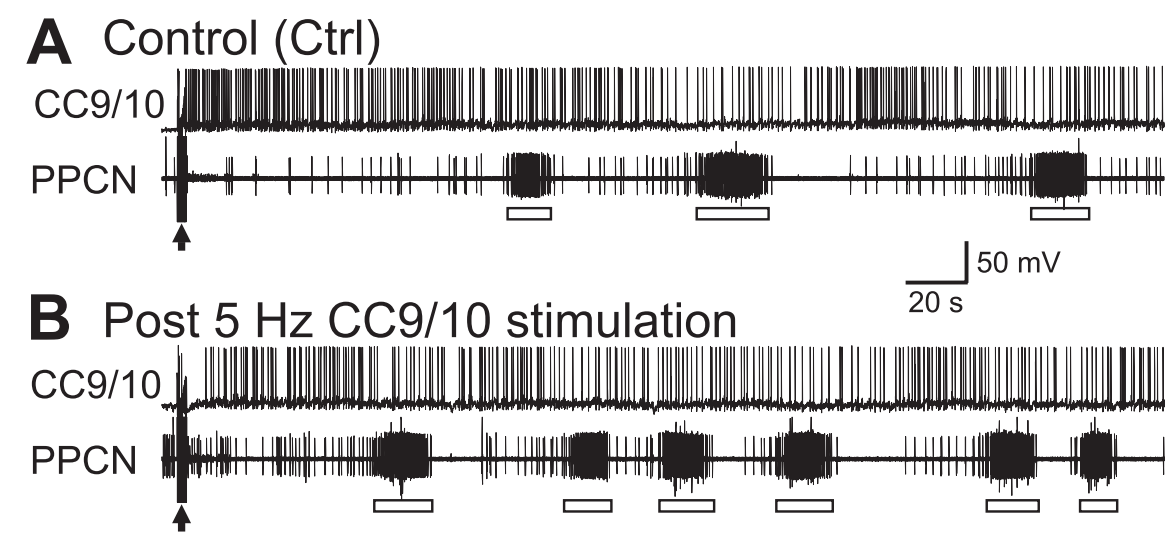

Figure 5. CC9/10 activity enhances subsequent locomotor motor programs elicited by stimulation of the $A_{4}$ nerve. $A, L_{0} 0-$ motor activity (open bars) elicited by $\mathrm{AT}_{4}$ stimulation (arrow) under control conditions was infrequent. $\boldsymbol{B}$, When $\mathrm{CC} / 10$ was stimulated at $5 \mathrm{~Hz}$ for 2 min before $\mathrm{AT}_{4}$ stimulation (arrow), locomotor activity was more frequent, and occurred after a shorter delay.

(Fredman and Jahan-Parwar, 1983). [In semi-intact preparations, bursting activity in PPCN is an accurate index of foot motor activity and locomotion (Jahan-Parwar and Fredman, 1978, 1980).] The P9 stimulation paradigm reliably induced locomotor activity in PPCN (cycle frequency, $0.044 \pm 0.004 \mathrm{~Hz}, n=9$ ) and spiking activity in CC9/10 (average frequency over a 2 min pe- riod, $1.57 \pm 0.14 \mathrm{~Hz}$, peak frequency over a 5 s period, $2.75 \pm 0.29 \mathrm{~Hz}, n=8$ ) (Fig. $3 A$, top).

We used a similar paradigm to stimulate the AT nerve $\left(\mathrm{AT}_{4}\right)$. Stimulation of this nerve also elicited locomotor activity (cycle frequency, $0.021 \pm 0.004 \mathrm{~Hz}, n=$ 8 ), but the cycle frequency was lower than that of programs elicited by $\mathrm{P} 9$ stimulation $\left(p<0.01, t=3.87\right.$, unpaired $t$ test). $\mathrm{AT}_{4}$ nerve stimulation also induced spiking in CC9/10 (mean frequency over a 2 min period, $1.01 \pm 0.11 \mathrm{~Hz}$, peak frequency over a $5 \mathrm{~s}$ period, $2.21 \pm 0.15 \mathrm{~Hz}, n=9$ ) (Fig. $3 B$, bottom). During a 2 min period immediately following $\mathrm{AT}_{4}$ stimulation, the average CC9/10 frequency was lower than following P9 stimulation ( $p<0.01, t=$ 3.15 , unpaired $t$ test). This was consistent with a lower cycle frequency of locomotor activity elicited by $\mathrm{AT}_{4}$ stimulation compared with $\mathrm{P} 9$ stimulation. In contrast, the peak CC9/10 frequency over a $5 \mathrm{~s}$ period was not significantly lower $(p=0.11, t=$ 1.69 , unpaired $t$ test), suggesting that the initial high firing frequency of CC9/10 is not an important determinant of locomotor activity since it persists for several minutes.

In a second series of experiments, we examined whether direct activation of CC9/10 can initiate locomotor activity. We stimulated single CC9/10 cells with short current pulses at $5 \mathrm{~Hz}$ and found that locomotor activity recorded in PPCN was present for as long as CC9/10 was stimulated. Locomotor activity usually persisted for 1-3 min at somewhat lower cycle frequencies after CC9/10 stimulation was terminated (Fig. $3 B$ ). In addition to stimulation at $5 \mathrm{~Hz}$, we also tested other frequencies. With a $2 \mathrm{~Hz}$ stimulation paradigm (see Fig. 7A), in 4 of 7 preparations, no locomotor activity was observed, and in the other 3 preparations, only one or two bursts were observed, suggesting that $2 \mathrm{~Hz}$ stimulation is close to threshold. Note that $2 \mathrm{~Hz}$ stimulation of a single CC $9 / 10$ cell is higher than the average frequency of individual CC9/10 cells recorded during nerve stimulation. This is expected because nerve stimulation will activate both CC9 and $\mathrm{CC10}$, and the combined activity of CC9 and CC10 would be above $2 \mathrm{~Hz}$. At 5 $\mathrm{Hz}$ and above, locomotor activity was observed in all preparations. When CC9/10 was stimulated at $5 \mathrm{~Hz}$ for $2 \mathrm{~min}$, the cycle frequency of locomotor activity was $0.037 \pm 0.004 \mathrm{~Hz}(n=10)$. This is lower than the frequency elicited by P9 stimulation, but higher than the frequency elicited by $\mathrm{AT}_{4}$ stimulation. We also calculated the locomotor activity cycle frequency during a $2 \mathrm{~min}$ period following CC9/10 stimulation $(0.021 \pm 0.002 \mathrm{~Hz})$. This was significantly lower than the frequency observed during 

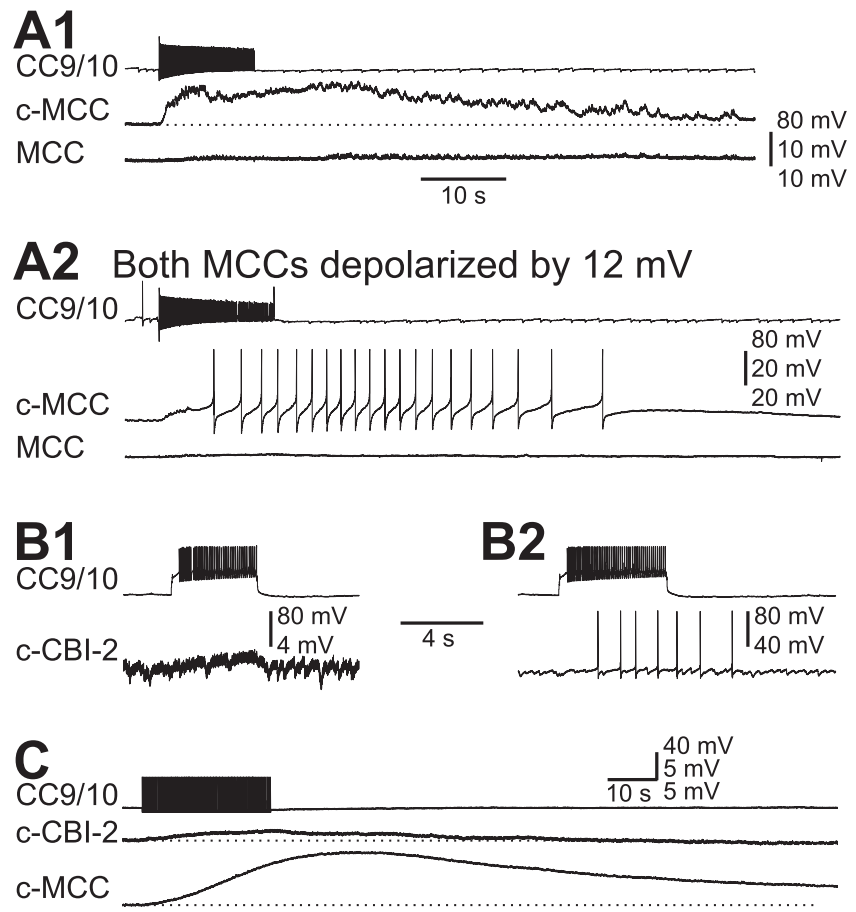

Figure 6. $\quad C(9 / 10$ elicits slow excitation in $\mathrm{MCC}$ and $\mathrm{CBI}-2$. ., , $C(9 / 10$ elicited slow EPSPs in the contralateral $\mathrm{MCC}(\mathrm{C}-\mathrm{MCC})$, but not in the ipsilateral MCC at resting potential $(\boldsymbol{A} \mathbf{A})$. When bilateral MCCs were each depolarized by $\sim 12 \mathrm{mV}$, stimulation of $C(9 / 10$ induced spike activity in the C-MCC (A2). Spikes of the C-MCC in $\boldsymbol{A} 2$ are clipped. $\boldsymbol{B}, C_{C}(9 / 10$ elicited a slow EPSP in the contralateral $\mathrm{CBI}-2(\mathrm{C}-\mathrm{CBI}-2)$, but did not trigger spiking when $\mathrm{CBI}-2$ was held at its resting potential (B1). When (BI-2 was depolarized by $25 \mathrm{mV}$, stimulation of $\mathrm{CC} 9 / 10$ triggered spikes in CBI-2 (B2). C, Stimulation of CC9/10 at $5 \mathrm{~Hz}$ elicited a slow EPSP in the C-MCC that developed gradually and persisted tens of seconds after the termination of $\mathrm{CC} 9 / 10$ stimulation. The slow EPSP in the C-CBI-2 was also present but was smaller and less persistent than the PSP in the MCC. Horizontal dotted lines: resting membrane potential. Recordings in $\boldsymbol{A}$ and $\boldsymbol{B}$ were made in normal saline. Recordings in C were made in high divalent saline.

CC9/10 stimulation ( $p<0.001, t=5.33$, paired $t$ test). Together, these data suggest that a higher activity level of CC9 and CC10 activity is associated with a higher cycle frequency of locomotor activity, and CC9 and CC10 may act as locomotion initiators.

Finally, since axons of CC9-10 project to the contralateral pedal ganglion, we examined synaptic connections from CC9/10 to two groups of pedal cells. The first group consists of a cluster of serotonergic modulatory neurons named the pedal serotonergic (PS) cluster (Fig. 4A) (Marinesco et al., 2004) that enhance contractions of foot and body wall muscles (McPherson and Blankenship, 1992). The somata of these neurons are relatively large and are typically located near the pedal commissure on both dorsal and ventral sides. Some of these neurons can be observed as two clusters of pigmented large neurons on the dorsal surface, named sectors Ia, Ib previously (Hening et al., 1979). The second group consists of neurons of sector II, on the dorsal surface, that contains several neurons which have been identified as foot motoneurons involved in locomotion (Hening et al., 1979). We found that CC9/10 synapses on many neurons in the PS cluster on both dorsal (i.e., sectors Ia and Ib) and ventral surfaces (20 of 24 neurons in 5 preparations). Most of the chemical connections appear to be monosynaptic as EPSPs followed presynaptic spikes one-for-one and persisted in high divalent saline (Fig. $4 B$ ). In at least one case, we found that CC9/10 had an electrical connection with a PS neuron. Furthermore, we verified that some neurons of PS cluster, which were postsynaptic to $\mathrm{CC} / 10$, were serotonergic using immunocytochemistry (Fig. $4 C$ ). In contrast, most neu- rons of sector II that we sampled had no connections from CC9/ 10. These data suggest that one function of CC9/10 is to provide excitation to serotonergic modulatory neurons of the pedal ganglion.

\section{Enhancement of nerve-evoked locomotor activity by CC9/10}

Having determined that CC9-10 are capable of initiating locomotion, we also sought to determine whether they can modulate locomotor activity that is initiated in some other manner. That this might be the case was suggested by the persistence observed in the experiments in which CC9/10 stimulation was used to induce activity. To test for a modulatory role we sought to determine whether prior stimulation of CC9-10 could enhance locomotor activity elicited by nerve stimulation. We selected the $\mathrm{AT}_{4}$ nerve for these experiments because $\mathrm{AT}_{4}$ evoked programs are weak (i.e., have a low cycle frequency) and are thus susceptible to further modulation. We also adjusted the voltage used to stimulate $\mathrm{AT}_{4}$ so that the cycle frequency under control conditions was below $0.03 \mathrm{~Hz}$. For preparations in which CC9/10 displayed robust spontaneous activity of $>2 \mathrm{~Hz}$, a small constant hyperpolarizing current $(<1 \mathrm{nA})$ was applied to decrease baseline activity. The $\mathrm{AT}_{4}$ nerve was stimulated once every 10-15 min. Under control conditions, we stimulated $\mathrm{AT}_{4}$ twice. Activity evoked after the second period of stimulation is shown in Figure $5 A$. We waited $7.5 \mathrm{~min}$, then stimulated CC9/10 at $5 \mathrm{~Hz}$ for $2 \mathrm{~min}$. Thirty seconds after termination of CC9/10 stimulation, we stimulated $\mathrm{AT}_{4}$ for the third time, and show activity evoked in this manner in Figure $5 B$ (post CC9/10 stimulation). Group data showed that cycle frequency of "post CC9/10" motor programs (0.024 \pm $0.002 \mathrm{~Hz}$ ) was significantly faster than cycle frequency of control programs $(0.014 \pm 0.002 \mathrm{~Hz})(p<0.05, t=4.49$, paired $t$ test, $n=5$ ). In addition, program latency (the time from the end of $\mathrm{AT}_{4}$ stimulation to the onset of the first burst in PPCN) of "post CC9/10" motor programs (40.5 $\pm 6.33 \mathrm{~s})$ was significantly shorter than program latency of control programs $(69.78 \pm 13.02$ s) $(p<0.05, t=2.97$, paired $t$ test, $n=5)$.

Because locomotor activity persists following $5 \mathrm{~Hz}$ stimulation of CC9/10, the effects of CC9/10 stimulation could be partly due to a summation of $\mathrm{CC} 9 / 10$ and $\mathrm{AT}_{4}$ stimulation. We therefore performed experiments as described above, but with $2 \mathrm{~Hz}$ stimulation of CC9/10 (instead of $5 \mathrm{~Hz}$ ). At $2 \mathrm{~Hz}, \mathrm{CC} 9 / 10$ stimulation does not normally elicit programs. Again prestimulation of CC9/10 enhanced the cycle frequency of locomotor activity elicited by $\mathrm{AT}_{4}$ stimulation (Control: $0.017 \pm 0.002 \mathrm{~Hz}$, vs post CC9/10: $0.022 \pm 0.002 \mathrm{~Hz} ; p<0.05, t=3.53$, paired $t$ test). In this case, however, the latency of locomotor activity following $\mathrm{AT}_{4}$ stimulation was not significantly shorter than in control preparations (Control: $32.52 \pm 3.17 \mathrm{~s}$, vs post CC9/10: $30.94 \pm$ $3.53 \mathrm{~s} ; p=0.63 ; t=0.52$, paired $t$ test). These data indicate that prestimulation of CC9/10 enhances locomotor activity elicited by nerve stimulation, and is effective even when the CC9/10 stimulation frequency does not drive motor programs.

In principle, at least two possible mechanisms could underlie the CC9/10 induced enhancement of $\mathrm{AT}_{4}$-elicited locomotor activity. First, prior stimulation of CC9/10 could enable $\mathrm{AT}_{4}$ to increase the CC9/10 firing frequency during motor activity and this increase in CC9/10 activity could be responsible for the higher cycle frequency of locomotor activity. Second, CC9/10 could enhance the excitability of the locomotor CPG in the pedal ganglion, which would allow $\mathrm{AT}_{4}$ to trigger locomotor activity with a higher cycle frequency. To distinguish between these two possibilities, we calculated the average frequency of CC9/10 firing following $\mathrm{AT}_{4}$ stimulation in control and experimental trials. We 
found that CC9/10 activity in response to $\mathrm{AT}_{4}$ stimulation did not increase in trials with prior CC9/10 stimulation. Instead, CC9/10 activity decreased, and this decrease was statistically significant with 5 $\mathrm{Hz}$ stimulation of CC9/10 (Control: $1.40 \pm 0.10 \mathrm{~Hz}$, vs post CC9/10: $0.85 \pm$ $0.08 \mathrm{~Hz} ; p<0.05, t=3.74$, paired $t$ test), but was not statistically significant with 2 $\mathrm{Hz}$ stimulation of CC9/10 (Control: $1.15 \pm 0.21 \mathrm{~Hz}$, vs post CC9/10: $0.92 \pm$ $0.22 \mathrm{~Hz} ; p=0.17 ; t=1.66$, paired $t$ test). These data support the idea that CC9/10 promotes nerve-evoked locomotor activity by enhancing the excitability of the pedal locomotor CPG.

\section{Interactions of the locomotor and feeding circuits}

The preceding sections show that CC9-10 are capable of initiating locomotion. CC9-10 also act as modulators of the locomotor circuit because CC9-10 activity can enhance generation of programs that are elicited by subsequent nerve stimulation. In the following experiments, we examined potential interactions of CC $9-10$ with the feeding circuit. Because CC9-10 are located in the cerebral ganglion, we examined potential synaptic interactions between CC9-10 and other cerebral cells (Rosen et al., 1991; Jing et al., 2004; Jing and Weiss, 2005; Koh and Weiss, 2007; Sasaki et al., 2007, 2008; Wu et al., 2007). We found that CC9-10 induced a slow depolarization in two feeding command neurons, CBI-2 $(n=5)$ and CBI-12 $(n=$ 3). Additionally, CC9-10 depolarized a neuromodulatory element of the feeding circuit, the serotonergic MCC $(n=23)$. Below we describe these inputs in more detail.

Activation of CC9/10 did not elicit a response in the ipsilateral MCC but did trigger a slow EPSP in the contralateral cell (Fig. 6). This slow potential assumed the form of a slow depolarization that persisted for tens of seconds after the stimulation of CC9/10 had been terminated. When the MCC was held at resting potentials (approximately $-67 \mathrm{mV}$ ), stimulation of CC9/10 typically did not trigger spiking (Fig. 6A1). However, when the holding potential of the MCC was increased, brief CC9/10 stimulation induced spiking that outlasted the period of stimulation (Fig. 6A2). The slow EPSP was observed in high divalent saline (Fig. 6C), indicating that the connection is monosynaptic. Recordings obtained in high divalent saline also showed that when CC9/10 was stimulated at $5 \mathrm{~Hz}$, slow EPSPs in the MCC developed gradually and
A1 $5 \mathrm{~Hz}$
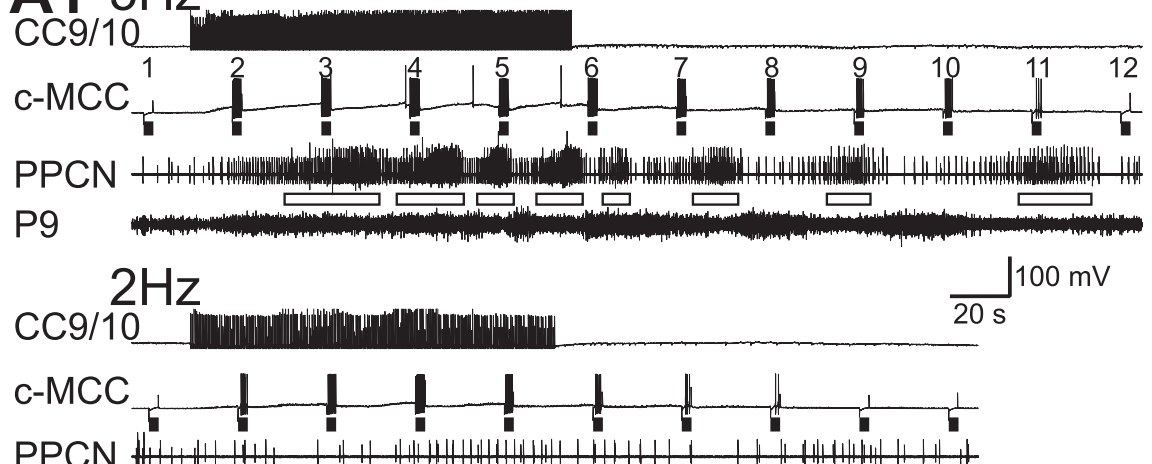

P9
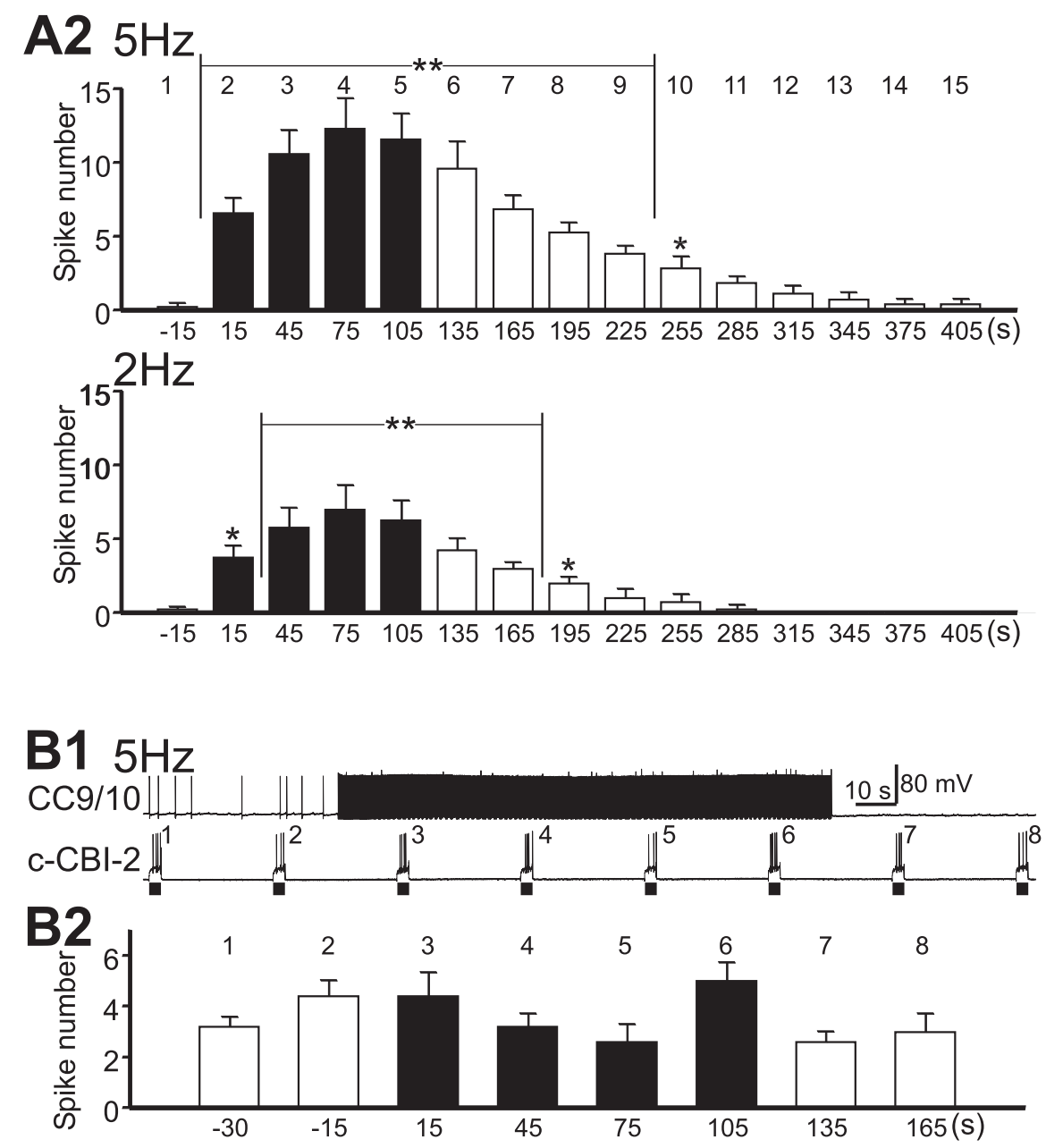

Figure 7. $\quad C C 9 / 10$ enhances MCC excitability but has little effect on CBI-2 excitability. A1, Stimulation of $\mathrm{CC} 9 / 10(5 \mathrm{~Hz}$, top) enhanced MCC excitability, and induced locomotor activity (open bars) in PPCN. Lower frequency stimulation of $\mathrm{CC} 9 / 10(2 \mathrm{~Hz}$, bottom) no longer induced locomotor activity, but still enhanced MCC excitability. MCC excitability was tested with $3 \mathrm{~s}$ current pulses (bars) applied every $30 \mathrm{~s}$, and the numbers above the $\mathrm{C}-\mathrm{MCC}$ denote test number. A2, Group data showing the effects of $C C 9 / 10$ on MCC excitability (measured by counting the number of MCC spikes evoked by $3 \mathrm{~s}$ current pulses) when $C(9 / 10$ was stimulated at $5 \mathrm{~Hz}\left(F_{(15,90)}=26.44, p<0.0001, n=7\right)$ and $2 \mathrm{~Hz}\left(F_{(10,30)}=11.61, p<0.0001, n=4\right)$. Numbers on the top of the graph correspond to the test numbers shown at part $A 1$. Post hoc tests [the number of $\mathrm{MCC}$ spikes at each test is compared with the number of spikes at test $1(-15 \mathrm{~s})$ ] with Dunnett corrections are as follows: ${ }^{*} p<0.05 ;{ }^{* *} p<0.01 . B 1$, Effects of stimulation of $\mathrm{CC} 9 / 10$ at $5 \mathrm{~Hz}$ on the excitability of $\mathrm{CBI}-2 . \mathrm{B2}$, Group data showing that $5 \mathrm{~Hz} \mathrm{CC} / 10$ stimulation had no significant effect on the excitability of $\mathrm{CBI}-2\left(F_{(7,32)}=2.10, p>0.05, n=5\right)$. Numbers on the top of the graph correspond to the test numbers shown at part $B 1$. Black bars in bar graphs are data obtained during stimulation of CC9/10. Error bars indicate SEM. 
persisted beyond CC9/10 stimulation. Thus, these data suggest that CC9/10 elicits slow excitation in the contralateral MCC, but that this excitation is not strong enough to directly activate the MCC. Rather, the slow excitation may act to enhance MCC excitability.

The slow excitation from CC9/10 to the contralateral CBI-2 appeared to be weak, and did not trigger spiking when CBI-2 was held at resting potential (Fig. 6B1). Activity was only evoked if CBI-2 was depolarized to within a few $\mathrm{mV}$ of its threshold before CC9/10 was activated (Fig. 6B2). A similar type of connection was observed between CC9/10 and CBI-12 (data not shown). The weak connection between CC9/10 and CBI-2 was present in high-divalent saline, suggesting that it is monosynaptic $(n=3)$ (Fig. $6 C)$. To compare the CC9/10 connection to CBI-2 to the connection with the MCC, we performed experiments in which we simultaneously recorded from all three cells. We found that the slow EPSP from CC9/10 to MCC was larger and more persistent than the slow EPSP from CC9/10 to $\mathrm{CBI}-2$ (Fig. 6C).

\section{Conditional and persistent activation of the MCC}

To formally evaluate the functional effects of CC9/10 on MCC excitability, we repeatedly applied $3 \mathrm{~s}$ depolarizing current pulses to the MCC that were either just below spike threshold or were just barely suprathreshold (i.e., only evoked 1-3 spikes). We then stimulated CC9/10 at various frequencies for 2 min to observe excitability changes. Figure $7 A$ shows an experiment in which we stimulated CC9/10 at both $5 \mathrm{~Hz}$ and $2 \mathrm{~Hz}$. In this experiment the MCC current pulses were subthreshold before CC9/10 stimulation. When $\mathrm{CC} 9 / 10$ was stimulated at $5 \mathrm{~Hz}$ spikes were evoked in the MCC and locomotor activity was induced (note the bursting activity in PPCN). Spiking in the MCC persisted for $>2$ min after CC9/10 stimulation. To determine whether the enhancement of MCC excitability depended on the induction of locomotor activity, we stimulated CC9/10 at $2 \mathrm{~Hz}$. We found that MCC excitability still increased, although locomotor activity was not present (as determined by the absence of bursting activity in the PPCN). Figure $7 B$ shows group data of the effects of stimulating CC9/10 at either $5 \mathrm{~Hz}\left(F_{(15,90)}=26.44\right.$, $p<0.0001, n=7)$ or $2 \mathrm{~Hz}\left(F_{(10,30)}=11.61, p<0.0001, n=\right.$ $4)$. At both frequencies, CC9/10 stimulation was effective in enhancing MCC excitability both during and after the stimulation. Two $\mathrm{Hz}$ stimulation produced smaller and less persistent effects on excitability than $5 \mathrm{~Hz}$ stimulation.

For the purpose of comparison, we also examined effects of CC9/10 stimulation on CBI-2 excitability. In contrast to the strong effects of CC9/10 stimulation on MCC excitability, stimulation of $\mathrm{CC} / 10$ at $5 \mathrm{~Hz}$ had no significant effects on CBI-2 excitability $\left(F_{(7,32)}=2.10, p>0.05, n=5\right)$ (Fig. $\left.7 B\right)$. This is consistent with the overall weak effects of CC9/10 on CBI-2 (Fig. 6C).

The enhancing effect of CC9/10 on MCC excitability is intriguing, because it indicates that effects are conditional. Thus, CC9/10 normally does not activate MCC by itself, a second input is necessary. A likely possibility would be that this input would be food related. A previously identified sensory neuron activated by food is the cerebral C2 neuron, which is histaminergic (Weinreich et al., 1975; Weinreich, 1977; McCaman and Weinreich, 1985; Weiss et al., 1986a) and nitregic (Jacklet, 1995; Koh and Jacklet, 1999), is a mechanosensory neuron (Weiss et al., 1986c), is rhythmically active during feeding behavior (Weiss et al., 1986b), and provides slow EPSPs onto the ipsilateral MCC. This network organization provides us an opportunity to examine potential interactions between the two modulatory pathways, both of which act on the MCC.

To characterize effects on the MCC when both $\mathrm{C} 2$ and CC9/10 were stimulated we performed the experiment shown in Figure 8. In Figure $8 A$, we show that when the MCC was at its resting potential, stimulation of CC9/10 at $5 \mathrm{~Hz}$ for $2 \mathrm{~min}$ did not induce significant spiking ( 3 spikes were evoked in the 2 min stimulation period, and no spikes were evoked thereafter). In Figure $8 B$, we stimulated C2 at $20 \mathrm{~Hz}$ for $3 \mathrm{~s}$ every $30 \mathrm{~s}$. C2 stimulation generated a slow EPSP in the MCC, but also failed to induce spiking. However when $\mathrm{C} 2$ and $\mathrm{CC} 9 / 10$ were coactivated spiking in the MCC was evoked. Significantly, even after the termination of CC9/10 stimulation, C2 stimulation retained its ability to elicit spikes in the MCC for a couple of minutes. Group data $(n=6)$ showed a significant difference ( $p<0.01, t=4.29$, paired $t$ test) between the number of spikes generated by the MCC in the $2 \mathrm{~min}$ period during which CC9/10 was stimulated alone $(6.33 \pm 1.91)$ vs when both CC9/10 and C2 were stimulated (40.67 \pm 9.48$)$. Similarly, group data also showed a significant difference $(p<$ $0.05, t=3.76$, paired $t$ test) between the number of spikes in the MCC during the 2 min period following CC9/10 stimulation when only CC9/10 was stimulated $(2.67 \pm 1.31)$ vs when both CC9/10 and C2 were stimulated (13.17 \pm 3.78$)$.

Experiments shown in Figure $3 \mathrm{~A}$ demonstrated that brief stimulation of two peripheral nerves, $\mathrm{P} 9$ and $\mathrm{AT}_{4}$, induced prolonged spike-activity in CC9/10. Interestingly, previous work has indicated that although P9 stimulation elicits persistent activity in many serotonergic neurons in the Aplysia CNS (compare Fig. 3 ), it does not trigger spiking in the serotonergic MCCs (Marinesco et al., 2004). Our data suggest, however, that MCC excitability may be increased. Specifically, we show that CC9 and CC10 are persistently activated by $\mathrm{P} 9$ and $\mathrm{AT}_{4}$ stimulation, and that CC9/10 activation modifies MCC excitability. [C2 is 
A
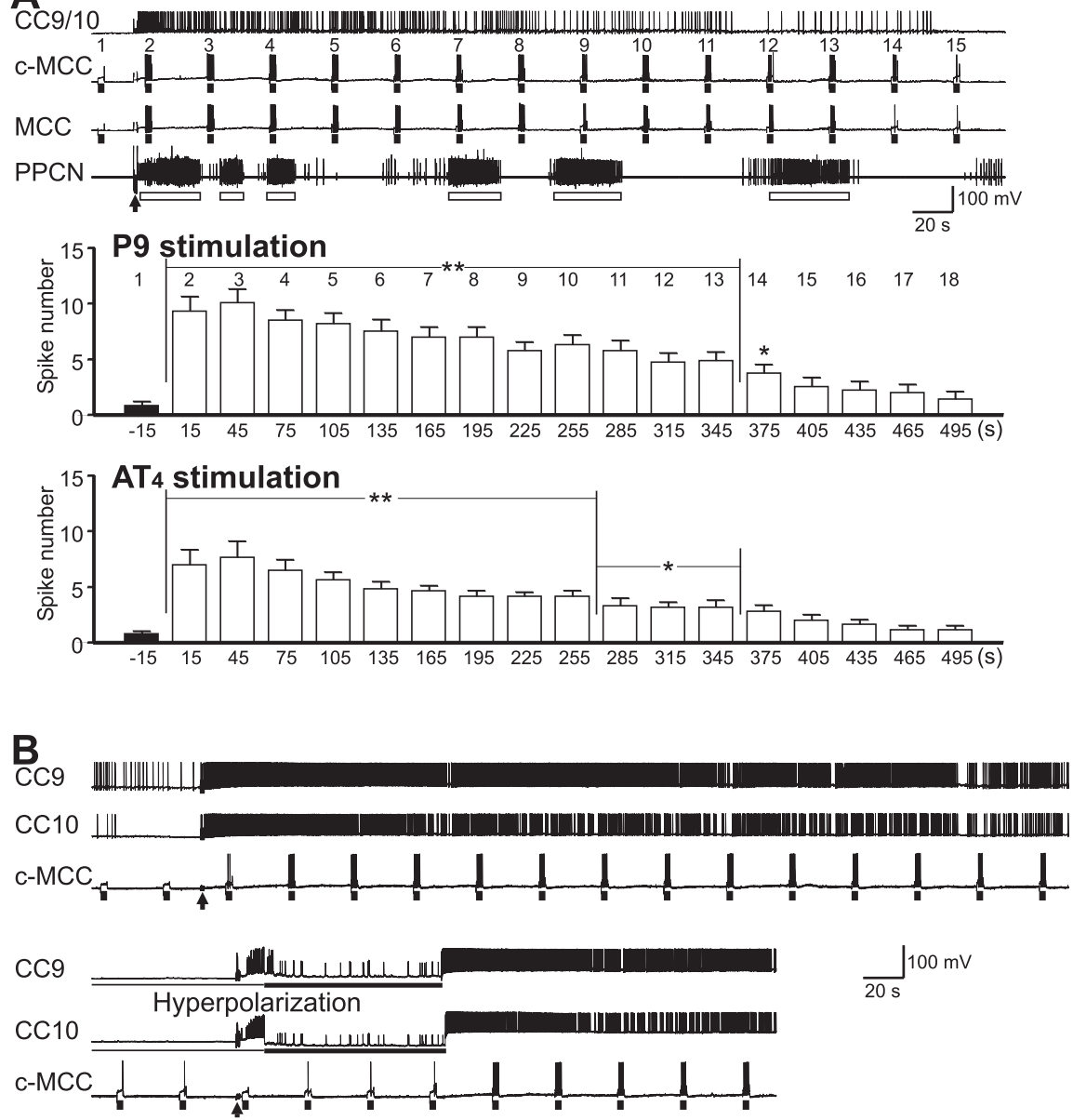

Figure 9. Briefnerve stimulation enhances $M C C$ excitability, an effect that appears to be largely mediated by $C(9 / 10 . A, A$ brief stimulation of $\mathrm{P9}$ (arrow) induced prolonged activity in $\mathrm{C}(9 / 10$ that was accompanied by locomotor activity (open bars) in PPCN as well as a bilateral increase in the excitability of the MCC. MCC excitability was tested with $3 \mathrm{~s}$ current pulses (bars) applied every $30 \mathrm{~s}$, and the numbers above the $\mathrm{C}-\mathrm{MCC}$ denote test number. Bar graph at the bottom: group data showing the effects of $\mathrm{P} 9$ and $\mathrm{AT}_{4}$ stimulation on MCC excitability. Numbers on the top of the graph correspond to test numbers above the recordings of the C-MCC. Posthoctests [the number of MCC spikes at each test is compared with the number of spikes at test $1(-15 \mathrm{~s})$ ] with Dunnett corrections are as follows: ${ }^{*} p<0.05 ;{ }^{* *} p<0.01$. Error bars indicate SEM. $\boldsymbol{B}$, An experiment that was similar to the one shown in $A$ but with recordings from both $\mathrm{C} C 9$ and $\mathrm{CC} 10$. Hyperpolarization of both $\mathrm{C} C 9$ and $\mathrm{CC} 10$ (bottom) largely prevented the increase in contralateral MCC excitability after P9 stimulation (arrows). When the hyperpolarization was released, $\mathrm{CC} 9$ and $\mathrm{CC} 10$ started firing and MCC excitability increased.

not persistently activated by $\mathrm{P} 9$ or $\mathrm{AT}_{4}$ stimulation (data not shown).] To determine whether MCC excitability is altered we performed the type of experiment shown in Figure 9A. We briefly stimulated P9 (Fig. 9A, top) and tested MCC excitability (with 3 s injections of depolarizing current pulses that were applied every $30 \mathrm{~s}$ ). Note that after P9 stimulation, the MCC was not active unless depolarized by 3 s current pulses used to test excitability, confirming previous findings. We did, however, find that MCC excitability was enhanced for prolonged periods after P9 stimulation. Similar results were obtained when $\mathrm{AT}_{4}$ was stimulated. Group data (Fig. 9A, bottom) indicated that MCC excitability was significantly enhanced up to 6 min after P9 $\left(F_{(17,136)}=20.20, p<0.0001, n=9\right)$ or $\mathrm{AT}_{4}$ $\left(F_{(17,85)}=14.53, p<0.0001, n=6\right)$ stimulation.

To characterize the contribution of CC9/10 activity to the enhancement of MCC excitability following nerve stimulation, we recorded from both CC9 and CC10 together with the contralateral MCC, and performed CC9 and CC10 hyperpolarization experiments. Under control conditions, brief stim- ulation of P9 induced persistent activity in both CC9 and CC10 with a concomitant increase in MCC excitability. However, when we hyperpolarized both CC9 and $\mathrm{CC} 10$ to prevent their firing, the increase in MCC excitability was largely abolished (Fig. 9B). Subsequently, when CC9 and CC10 were released from hyperpolarization, both cells resumed firing, and MCC excitability was again increased. For the purpose of statistical analysis, we calculated the ratios of the maximum number of MCC spikes evoked during a $3 \mathrm{~s}$ pulse after P9 stimulation to the number of spikes evoked before P9 stimulation. The ratio was taken as a measure of the change in MCC excitability. The average ratio in control conditions was $7.25 \pm 1.65$; but decreased to $2.44 \pm 1.19$ when CC9 and CC10 were hyperpolarized. The differences in the average ratio under control vs experimental conditions are statistically significant $(p<0.05, t=4.35, n=$ 4 , paired $t$ test). These data suggest that, to a large extent, CC9/10 may be responsible for the increase in MCC excitability that is observed after P9 nerve stimulation.

\section{Sensory activation}

To explore the functional contexts in which the CC9-10 enhancement of MCC excitability may operate, we examined CC9-10 activity in response to sensory stimuli in semi-intact preparations. In these preparations, peripheral structures (head and/or tails) are attached to the CNS through peripheral nerves, and physiologically relevant stimuli can be peripherally applied. For example, feeding can be induced by food application.

CC9/10 was active in 7 of 10 trials (3 different preparations) in which we elicited feeding via a food stimulus. In 3 of these 7 trials, CC9/10 was not active before seaweed application, but became active $(0.81 \pm$ $0.06 \mathrm{~Hz}, n=3$ ) after the seaweed was applied and during ingestion of food (Fig. 10A, top). In the other 4 trials, CC9/10 was spontaneously active, and application of seaweed did not further increase its firing rate $(0.83 \pm 0.11 \mathrm{~Hz}, n=4)$ (Fig. $10 \mathrm{~A}$, bottom). As expected food application also activated the MCC.

In addition, in these 3 preparations, we tested the effects of tactile (tentacle touch) and noxious stimuli (tail or tentacle pinch) on CC9/10 activity. Tactile stimuli generally were not effective in activating CC9/10 (data not shown). In contrast, tentacle and tail pinch were effective. Afferent activity generated by pinching the tentacle, and believed to be carried partly by the $\mathrm{AT}_{4}$ nerve, activated CC9/10 $(1.09 \pm 0.21 \mathrm{~Hz}, n=6)$ and depolarized the MCC (Fig. 10 B1). Similarly, pinching the tail, which is connected with the pedal ganglion through $\mathrm{P} 9$, also activated CC9/10 $(1.01 \pm 0.12 \mathrm{~Hz}, n=5)$ (Fig. 10 B2). In the example shown (Fig. 10 B2), we also tested MCC excitability by injecting 3 s depolarizing current pulses into the MCC every $30 \mathrm{~s}$. Tail pinch also 
enhanced MCC excitability, as demonstrated by the ability of depolarizing current pulses to elicit a larger number of spikes in the MCC after the tail pinch $(n=2)$.

\section{Discussion}

In addition to eliciting specific behaviors, a stimulus can induce an arousal state that persists after the stimulus is no longer present and manifests itself as an increased probability, strength, and/or speed of more than one behavior (Hebb, 1949). Early studies (Moruzzi and Magoun, 1949) suggested that arousal might be mediated by a central system that acts in a generalized manner to indiscriminately affect a broad spectrum of behaviors. Subsequent work has indicated that arousal is not mediated by a single centralized system. Instead, it results from the activation of multiple systems that may have differing ranges of actions (Vanderwolf and Robinson, 1981; Weiss et al., 1982; Robbins and Everitt, 1995; Pfaff, 2006). Some forms of arousal enhance one subset of behaviors and inhibit conflicting behaviors (Kupfermann, 1974). Circuitry mediating this type of interaction can be relatively simple, e.g., antagonistic behaviors can be suppressed via the inhibition of command and/or CPG neurons (Kovac and Davis, 1977; Edwards, 1991; Norekian and Satterlie, 1996; Shaw and Kristan, 1997; Jing and Gillette, 2000). Other forms of arousal are interesting, however, in that they can increase the probability of evoking two potentially conflicting behaviors (Rowell, 1961; Barfield and Sachs, 1968; Antelman and Szechtman, 1975). We studied Aplysia interneurons activated during the latter type of arousal, i.e., cells activated by mildly noxious stimuli. These stimuli induce locomotion and increase the likelihood that biting responses will be observed when animals are exposed to food (Kupfermann and Weiss, 1981). Feeding conflicts with locomotion because when consummatory feeding occurs, locomotion generally stops (Kupfermann, 1974; Gillette and Jing, 2001).

We demonstrated that cells activated by alerting/noxious stimuli include the serotonergic CC9-10 cells. Previous studies have demonstrated that brief shocks of the tail nerve persistently activate many serotonergic neurons that are widely distributed in the Aplysia CNS (Marinesco et al., 2004). The activation patterns of CC9-10 in response to nerve shock are qualitatively similar to those of other serotonergic neurons. We also showed that CC -10 are activated by food, tentacle and tail pinch, and by brief shocks of a head-innervating nerve. Thus, CC $9-10$ are broadly activated and their responses are not site-specific.

CC9-10 differ from most of the other serotonergic neurons in the pedal and abdominal ganglia, in that they affect activity in multiple motor networks. With the exception of CC3(CB1) (Mackey et al., 1989; Xin et al., 2001), actions of other identified cells in Aplysia are limited to individual motor networks (McPherson and Blankenship, 1992) (Fig. 11). In fact, CC9-10 and CC3 may contribute to the persistent activation of serotonergic modulatory neurons in the pedal and abdominal ganglia, respectively, through their preferential actions in these two ganglia (Fig. 4) (Xin et al., 2001; Marinesco et al., 2004). We showed that CC9-10 impact both locomotor and feeding networks.

In the context of locomotion, our data suggest that CC9-10 serve two functions. First, CC9-10 directly drive locomotor activity in a frequency-dependent manner. Importantly, locomotor activity outlasts CC9-10 stimulation. Moreover, a higher CC9-10 activity level during programs elicited by $\mathrm{P}$ vs $\mathrm{AT}_{4}$ stimulation is associated with a faster cycle frequency of locomotor activity. Together, these findings may account for the behavioral findings that serotonin promotes locomotion (Palovcik et al., 1982; Mackey and Carew, 1983). Notably, CC9-10 and type I command neurons for locomotion identified earlier (Fredman and Jahan-Parwar, 1983) are similar in their spiking pattern, axonal projections, locations, and roles in locomotion, but CC9-10 are electrically coupled whereas electrical coupling was not found in type I neurons. However, the type I neurons might possibly be coupled but this coupling was masked by the frequent spontaneous PSPs in normal saline. Other comparisons are not possible since there are no dye fills or immunocytochemical studies for the type I neurons. It is therefore likely but not certain that the two cell types are the same. CC9-10 may also be similar to type CN2 and/or CN4 command-like neurons that drive swimming in 


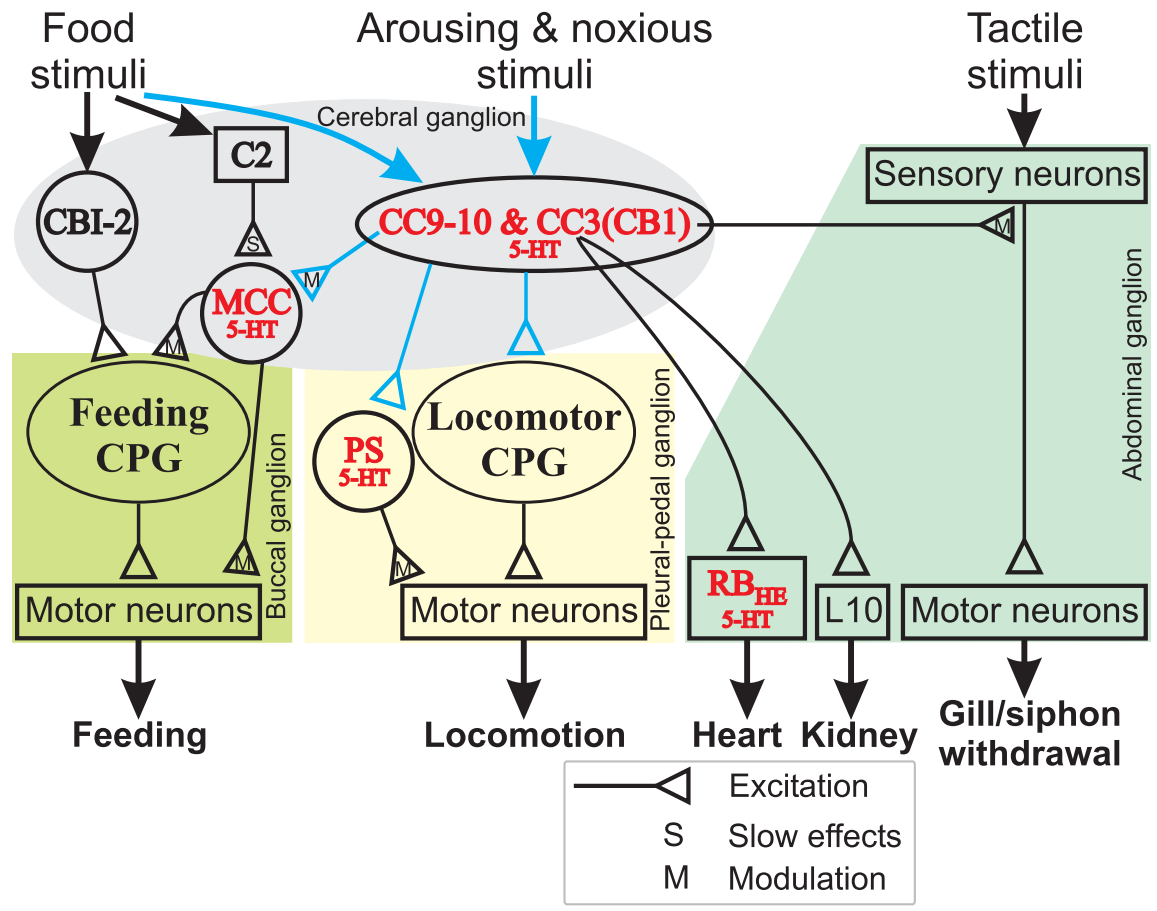

Figure 11. A schematic diagram illustrating a central role of serotonergic interneurons, $C(9-10$ and $C(3(C B 1)$ in a form of arousal elicited by noxious stimuli in Aplysia. CC3(CB1) data summarize previous work (Mackey et al., 1989; Xin et al., 2001; Marinesco et al., 2004). C(9-10 data are from this study, and are shown in blue. All serotonergic neurons (5-HT) are shown in red. The approximate ganglionic locations of the neural elements for different networks are also indicated. CC9-10 and CC3(CB1) constitute a group of heterogeneous serotonergic interneurons that are activated at different levels by a variety of stimuli. These neurons enhance the activity of various behavioral networks through fast excitatory and slow modulatory actions. CC9-10 and CC3(CB1) may preferentially act on the neurons in the pedal vs abdominal ganglia (e.g., the serotonergic PS vs RB neurons, respectively). C 9 and CC10 function to initiate locomotor activity. They enhance MCC excitability, thus promoting feeding in a manner that is conditional on the presence of food-related inputs (e.g., via food induced activation of (2). CC3(CB1) mediates presynaptic facilitation of sensory neurons and thus contributes to sensitization of gill and siphon withdrawal. $C(3)(C B 1)$ also excites the serotonergic heart excitor $\left(\mathrm{RB}_{\mathrm{HE}}\right)$ and $\mathrm{L} 10$ that promotes opening of the renal pore.

Aplysia brasiliana (Gamkrelidze et al., 1995). It is, however, unknown whether $\mathrm{CN} 2 / \mathrm{CN} 4$ are serotonergic.

Second, CC9-10 serve a modulatory role. CC9-10 promote $\mathrm{AT}_{4}$-elicited locomotor programs and effects on cycle frequency are observed even when CC9-10 are stimulated at frequencies subthreshold for motor program induction. Additionally, CC9-10 are likely homologs of the DSI2-3 cells in Tritonia (Getting, 1981; Katz et al., 1994; McClellan et al., 1994) and the As2-3 cells in Pleurobranchaea (Jing and Gillette, 1999, 2003). The DSI2-3 and As2-3 cells are similar serotonergic neurons that are part of the CPGs that mediate escape swimming in Tritonia and Pleurobranchaea. Extensive studies have shown that DSI2-3 neurons exert modulatory effects on synaptic output (Katz et al., 1994) and spike adaptation (Katz and Frost, 1997) of other swimming CPG interneurons.

Furthermore, CC9-10 act as extrinsic modulators of the feeding network by promoting MCC activity. This is reminiscent of earlier findings in Pleurobranchaea (Jing and Gillette, 2000) where As2-3 excite the MCC homolog, the metacerebral giant (MCG) neuron. Functional consequences of the activation of As2-3 and CC9-10 may differ somewhat. In Pleurobranchaea, As2-3 elicit fast monosynaptic EPSPs in the MCG, and they routinely induce MCG spiking, potentially contributing to spontaneous MCG firing (Gillette and Davis, 1977). In contrast, the effects of CC9-10 on the feeding network are conditional. That is, CC9-10 do not directly activate the MCCs. Instead, they increase MCC excitability so that responses to afferent (i.e., C2 mediated) input are enhanced. Notably, the CC9-10 induced depolarization may act in concert with the intrin- sic anomalous rectification of the MCC (Kandel and Tauc, 1966; Weiss and Kupfermann, 1976) to further enhance the EPSPs that C2 evokes. C2 (Weiss et al., 1986b) is activated when food contacts the mouth and remains active as feeding progresses. Thus, the effects of CC9-10 on the MCCs are not manifested unless another input is present (e.g., food). Importantly, the MCCs modulate feeding (Proekt and Weiss, 2003), but do not directly evoke it (Weiss et al., 1979).

From an evolutionary perspective, in addition to As2-3 and DSI2-3, similar cerebral serotonergic neurons are found in a number of mollusks, e.g., Clione (Panchin et al., 1995; Satterlie and Norekian, 1996) and nudibranchs (Tian et al., 2006; Newcomb and Katz, 2007). One common function may be their roles in locomotion. In Clione, locomotion occurs spontaneously but cerebral serotonergic neurons regulate locomotor speed. More dramatically, both DSI2-3 (Popescu and Frost, 2002) and CC9-10 drive locomotion, although the ciliary locomotion of Tritonia is radically different from the pedal locomotor waves of the Aplysia foot. Other commonality includes their central roles in organizing loosely coupled groups of serotonergic neurons in arousal in Aplysia, Pleurobranchaea (Jing and Gillette, 2000), and perhaps in Clione (Satterlie and Norekian, 1996). These observations suggest conservation of a significant part of central neuronal circuits in the face of extreme diversification of body form, diet and ecological niche (Gillette and Jing, 2001; Newcomb and Katz, 2007). The conservation could be a significant contributor to the success of the phylum and its radiation.

\section{Conclusions}

Food-induced arousal in Aplysia is mediated by the serotonergic MCCs (Weiss et al., 1978; Rosen et al., 1989; Proekt and Weiss, 2003) and peptidergic feeding interneurons (Morgan et al., 2000; Koh et al., 2003; Proekt et al., 2004, 2007). Here, we demonstrate that CC9-10 function as both initiators and modulators of locomotion. Moreover, CC9-10 avoid response conflict through conditional activation of the feeding network. Notably, CC9-10 do not play a major role in food-induced arousal because of their low level of activity during feeding. Instead, because CC9-10 are activated strongly by noxious/alerting stimulus, such as tail pinch and shock, they would promote locomotory behaviors immediately following the stimulus. Moreover, when food is present in a location with some delay (e.g., after locomotion), CC9-10 would promote feeding through their persistent conditional activation of the MCC. This is consistent with the behavioral observation that tail pinch can promote both locomotion and feeding (Kupfermann and Weiss, 1981). Our findings are also consistent with the notion that sequences in which behaviors are generated are threshold-dependent (Gillette et al., 2000). At rest, the feeding threshold is high but noxious stimuli readily induce locomotion because CC9-10 directly activate locomotion. After a period of locomotion, the feeding threshold may be lowered by CC $9-10$ conditionally activating the feeding network and thereby promoting, in a latent manner, food-evoked feeding. 
Thus, different forms of arousal are mediated by distinct but partially overlapping sets of neuronal elements that are defined by their response to external or internal stimuli that trigger specific forms of arousal, by their activity pattern that ensues, and by their specific outputs to different motor networks. In vertebrates, a variety of neuronal elements with different transmitter phenotypes similarly mediate various aspects of arousal (Robbins and Everitt, 1995; Pfaff, 2006) and thereby contribute to decision making (Doya, 2008). These transmitter systems include the raphe serotonergic neurons (Jacobs and Fornal, 1997; Gillette, 2006) and the locus ceruleus-norepinephrine neurons (Berridge and Waterhouse, 2003; Aston-Jones and Cohen, 2005). It is therefore worth exploring whether the relatively simple mechanisms present in Aplysia are also exploited by the more complex nervous systems of vertebrates.

\section{References}

Antelman SM, Szechtman H (1975) Tail pinch induces eating in sated rats which appears to depend on nigrostriatal dopamine. Science 189:731-733.

Aston-Jones G, Cohen JD (2005) An integrative theory of locus coeruleusnorepinephrine function: adaptive gain and optimal performance. Annu Rev Neurosci 28:403-450.

Barfield RJ, Sachs BD (1968) Sexual behavior: stimulation by painful electrical shock to skin in male rats. Science 161:392-393.

Berridge CW, Waterhouse BD (2003) The locus coeruleus-noradrenergic system: modulation of behavioral state and state-dependent cognitive processes. Brain Res Brain Res Rev 42:33-84.

Brembs B, Baxter DA, Byrne JH (2004) Extending in vitro conditioning in Aplysia to analyze operant and classical processes in the same preparation. Learn Mem 11:412-420.

Doya K (2008) Modulators of decision making. Nat Neurosci 11:410-416.

Edwards DH (1991) Mutual inhibition among neural command systems as a possible mechanism for behavioral choice in crayfish. J Neurosci 11:1210-1223.

Eisenstadt M, Goldman JE, Kandel ER, Koike H, Koester J, Schwartz JH (1973) Intrasomatic injection of radioactive precursors for studying transmitter synthesis in identified neurons of Aplysia californica. Proc Natl Acad Sci U S A 70:3371-3375.

Fickbohm DJ, Lynn-Bullock CP, Spitzer N, Caldwell HK, Katz PS (2001) Localization and quantification of 5-hydroxytryptophan and serotonin in the central nervous systems of Tritonia and Aplysia. J Comp Neurol 437:91-105.

Fredman SM, Jahan-Parwar B (1980) Role of pedal ganglia motor neurons in pedal wave generation in Aplysia. Brain Res Bull 5:179-193.

Fredman SM, Jahan-Parwar B (1983) Command neurons for locomotion in Aplysia. J Neurophysiol 49:1092-1117.

Furukawa Y, Nakamaru K, Wakayama H, Fujisawa Y, Minakata H, Ohta S, Morishita F, Matsushima O, Li L, Romanova E, Sweedler JV, Park JH, Romero A, Cropper EC, Dembrow NC, Jing J, Weiss KR, Vilim FS (2001) The enterins: a novel family of neuropeptides isolated from the enteric nervous system and CNS of Aplysia. J Neurosci 21:8247-8261.

Gamkrelidze GN, Laurienti PJ, Blankenship JE (1995) Identification and charaterization of cerebral ganglion neurons that induce swimming and modulate swim-related pedal ganglion neurons in Aplysia brasiliana. J Neurophysiol 74:1444-1462.

Getting PA (1981) Mechanisms of pattern generation underlying swimming in Tritonia. I. Neuronal network formed by monosynaptic connections J Neurophysiol 46:65-79.

Gillette R (2006) Evolution and function in serotonergic systems. Integr Comp Biol 46:838-846.

Gillette R, Davis WJ (1977) The role of the metacerebral giant neurone in the feeding behaviour of Pleurobranchaea. J Comp Physiol 116:129-159.

Gillette R, Jing J (2001) The role of the escape swim motor network in the organization of behavioral hierarchy and arousal in Pleurobranchaea. Am Zool 41:983-992.

Gillette R, Huang RC, Hatcher N, Moroz LL (2000) Cost-benefit analysis potential in feeding behavior of a predatory snail by integration of hunger, taste, and pain. Proc Natl Acad Sci U S A 97:3585-3590.

Hebb DO (1949) Organization of behavior. New York: Wiley.
Hening WA, Walters ET, Carew TJ, Kandel ER (1979) Motorneuronal control of locomotion in Aplysia. Brain Res 179:231-253.

Jacklet JW (1995) Nitric oxide is used as an orthograde cotransmitter at identified histaminergic synapses. J Neurophysiol 74:891-895.

Jacobs BL, Fornal CA (1997) Serotonin and motor activity. Curr Opin Neurobiol 7:820-825.

Jahan-Parwar B, Fredman SM (1978) Control of pedal and parapodial movements in Aplysia. I. Proprioceptive and tactile reflexes. J Neurophysiol 41:600-608.

Jahan-Parwar B, Fredman SM (1979) Neural control of locomotion in Aplysia: role of the central ganglia. Behav Neural Biol 27:39-58.

Jahan-Parwar B, Fredman SM (1980) Motor program for pedal waves during Aplysia locomotion is generated in the pedal ganglia. Brain Res Bull 5:169-177.

Jing J, Gillette R (1999) Central pattern generator for escape swimming in the notaspid sea slug Pleurobranchaea californica. J Neurophysiol 81:654-667.

Jing J, Gillette R (2000) Escape swim network interneurons have diverse roles in behavioral switching and putative arousal in Pleurobranchaea. J Neurophysiol 83:1346-1355.

Jing J, Gillette R (2003) Directional avoidance turns encoded by single interneurons and sustained by multifunctional serotonergic cells. J Neurosci 23:3039-3051.

Jing J, Weiss KR (2001) Neural mechanisms of motor program switching in Aplysia. J Neurosci 21:7349-7362.

Jing J, Weiss KR (2005) Generation of variants of a motor act in a modular and hierarchical motor network. Curr Biol 15:1712-1721.

Jing J, Vilim FS, Wu JS, Park JH, Weiss KR (2003) Concerted GABAergic actions of Aplysia feeding interneurons in motor program specification. J Neurosci 23:5283-5294.

Jing J, Cropper EC, Hurwitz I, Weiss KR (2004) The construction of movement with behavior-specific and behavior-independent modules. J Neurosci 24:6315-6325.

Jing J, Vilim FS, Horn CC, Alexeeva V, Hatcher NG, Sasaki K, Yashina I, Zhurov Y, Kupfermann I, Sweedler JV, Weiss KR (2007) From hunger to satiety: reconfiguration of a feeding network by Aplysia neuropeptide Y. J Neurosci 27:3490-3502.

Kabotyanski EA, Baxter DA, Cushman SJ, Byrne JH (2000) Modulation of fictive feeding by dopamine and serotonin in Aplysia. J Neurophysiol 83:374-392.

Kalén P, Rosegren E, Lindvall O, Björklund A (1989) Hippocampal noradrenaline and serotonin release over 24 hours as measured by the dialysis technique in freely moving rats: correlation to behavioural activity state, effect of handling and tail-pinch. Eur J Neurosci 1:181-188.

Kandel ER, Tauc L (1966) Anomalous rectification in the metacerebral giant cells and its consequences for synaptic transmission. J Physiol 183:287-304.

Katz PS, Frost WN (1997) Removal of spike frequency adaptation via neuromodulation intrinsic to the Tritonia escape swim central pattern generator. J Neurosci 17:7703-7713.

Katz PS, Getting PA, Frost WN (1994) Dynamic neuromodulation of synaptic strength intrinsic to a central pattern generator circuit. Nature 367:729-731.

Koh HY, Jacklet JW (1999) Nitric oxide stimulates cGMP production and mimics synaptic responses in metacerebral neurons of Aplysia. J Neurosci 19:3818-3826.

Koh HY, Weiss KR (2007) Activity-dependent peptidergic modulation of the plateau-generating neuron B64 in the feeding network of Aplysia. J Neurophysiol 97:1862-1867.

Koh HY, Vilim FS, Jing J, Weiss KR (2003) Two neuropeptides colocalized in a command-like neuron use distinct mechanisms to enhance its fast synaptic connection. J Neurophysiol 90:2074-2079.

Kovac MP, Davis WJ (1977) Behavioral choice: neurophysiological mechanism in Pleurobranchaea. Science 198:632-634.

Kupfermann I (1974) Feeding behavior in Aplysia: a simple system for the study of motivation. Behav Biol 10:1-26.

Kupfermann I, Weiss KR (1981) Tail pinch and handling facilitate feeding behavior in Aplysia. Behav Neural Biol 32:126-132.

Kupfermann I, Weiss KR (1982) Activity of an identified serotonergic neuron in free moving Aplysia correlates with behavioral arousal. Brain Res 241:334-337.

Lechner HA, Baxter DA, Byrne JH (2000) Classical conditioning of feeding in Aplysia: II. Neurophysiological correlates. J Neurosci 20:3377-3386. 
Longley RD, Longley AJ (1986) Serotonin immunoreactivity of neurons in the gastropod Aplysia californica. J Neurobiol 17:339-358.

Mackey S, Carew TJ (1983) Locomotion in Aplysia: triggering by serotonin and modulation by bag cell extract. J Neurosci 3:1469-1477.

Mackey SL, Kandel ER, Hawkins RD (1989) Identified serotonergic neurons LCB1 and RCB1 in the cerebral ganglia of Aplysia produce presynaptic facilitation of siphon sensory neurons. J Neurosci 9:4227-4235.

Marinesco S, Kolkman KE, Carew TJ (2004) Serotonergic modulation in Aplysia. I. Distributed serotonergic network persistently activated by sensitizing stimuli. J Neurophysiol 92:2468-2486.

McCaman RE, Weinreich D (1985) Histaminergic synaptic transmission in the cerebral ganglion of Aplysia. J Neurophysiol 53:1016-1037.

McClellan AD, Brown GD, Getting PA (1994) Modulation of swimming in Tritonia: excitatory and inhibitory effects of serotonin. J Comp Physiol [A] 174:257-266.

McPherson DR, Blankenship JE (1992) Neuronal modulation of foot and body-wall contractions in Aplysia californica. J Neurophysiol 67:23-28.

McPherson DR, Katz PS (2001) Identification of serotonergic cerebral neurons that project to the pedal ganglia in Aplysia californica. Soc Neurosci Abstr 27:934.10.

Morgan PT, Perrins R, Lloyd PE, Weiss KR (2000) Intrinsic and extrinsic modulation of a single central pattern generating circuit. J Neurophysiol 84:1186-1193.

Moruzzi G, Magoun HW (1949) Brain stem reticular formation and activation of the EEG. Electroencephalogr Clin Neurophysiol 1:455-473.

Mozzachiodi R, Lechner HA, Baxter DA, Byrne JH (2003) In vitro analog of classical conditioning of feeding behavior in Aplysia. Learn Mem 10:478-494.

Newcomb JM, Katz PS (2007) Homologues of serotonergic central pattern generator neurons in related nudibranch molluscs with divergent behaviors. J Comp Physiol A Neuroethol Sens Neural Behav Physiol 193:425-443.

Newcomb JM, Fickbohm DJ, Katz PS (2006) Comparative mapping of serotonin-immunoreactive neurons in the central nervous systems of nudibranch molluscs. J Comp Neurol 499:485-505.

Nolen TG, Carew TJ (1994) Ontogeny of serotonin-immunoreactive neurons in juvenile Aplysia californica -implications for the development of learning. Behav Neural Biol 61:282-295.

Norekian TP, Satterlie RA (1996) Cerebral serotonergic neurons reciprocally modulate swim and withdrawal neural networks in the mollusk Clione limacina. J Neurophysiol 75:538-546.

Ono JK, McCaman RE (1984) Immunocytochemical localization and direct assays of serotonin-containing neurons in Aplysia. Neuroscience 11:549-560.

Palovcik RA, Basberg BA, Ram JL (1982) Behavioral state changes induced in Pleurobranchaea and Aplysia by serotonin. Behav Neural Biol 35:383-394.

Panchin YV, Popova LB, Deliagina TG, Orlovsky GN, Arshavsky YI (1995) Control of locomotion in marine mollusk Clione limacina. VIII. Cerebropedal neurons. J Neurophysiol 73:1912-1923.

Pfaff DW (2006) Brain arousal and information theory: neural and genetic mechanisms. Cambrige, MA: Harvard UP.

Popescu IR, Frost WN (2002) Highly dissimilar behaviors mediated by a multifunctional network in the marine mollusk Tritonia diomedea. J Neurosci 22:1985-1993.

Proekt A, Weiss KR (2003) Convergent mechanisms mediate preparatory states and repetition priming in the feeding network of Aplysia. J Neurosci 23:4029-4033.

Proekt A, Brezina V, Weiss KR (2004) Dynamical basis of intentions and expectations in a simple neuronal network. Proc Natl Acad Sci U S A 101:9447-9452.

Proekt A, Jing J, Weiss KR (2007) Multiple contributions of an inputrepresenting neuron to the dynamics of the Aplysia feeding network. J Neurophysiol 97:3046-3056.

Robbins TW, Everitt BJ (1995) Arousal systems and attention. In: The cognitive neuroscience (Gazzaniga MS, ed), pp 703-720. Cambridge, MA: MIT.

Rosen SC, Weiss KR, Goldstein RS, Kupfermann I (1989) The role of a modulatory neuron in feeding and satiation in Aplysia: effects of lesioning of the serotonergic metacerebral cells. J Neurosci 9:1562-1578.

Rosen SC, Teyke T, Miller MW, Weiss KR, Kupfermann I (1991) Identification and characterization of cerebral-to-buccal interneurons implicated in the control of motor programs associated with feeding in Aplysia. J Neurosci 11:3630-3655.
Rowell TE (1961) Displacement grooming in the Chaffinch. Anim Behav 9:38-63.

Sasaki K, Due MR, Jing J, Weiss KR (2007) Feeding CPG in Aplysia directly controls two distinct outputs of a compartmentalized interneuron that functions as a CPG element. J Neurophysiol 98:3796-3801.

Sasaki K, Jing J, Due MR, Weiss KR (2008) An input-representing interneuron regulates spike timing and thereby phase switching in a motor network. J Neurosci 28:1916-1928.

Satterlie RA, Norekian TP (1995) Serotonergic modulation of swimming speed in the pteropod mollusc Clione limacina. III. Cerebral neurons. J Exp Biol 198:917-930.

Satterlie RA, Norekian TP (1996) Modulation of swimming speed in the pteropod mollusc, Clione limacina: role of a compartmental serotonergic system. Invert Neurosci 2:157-165.

Shaw BK, Kristan WB Jr (1997) The neuronal basis of the behavioral choice between swimming and shortening in the leech: control is not selectively exercised at higher circuit levels. J Neurosci 17:786-795.

Sudlow LC, Jing J, Moroz LL, Gillette R (1998) Serotonin-immunoreactivity in the central nervous system of the marine molluscs Pleurobranchaea californica and Tritonia diomedea. J Comp Neurol 395:466-480.

Sweedler JV, Li L, Rubakhin SS, Alexeeva V, Dembrow NC, Dowling O, Jing J, Weiss KR, Vilim FS (2002) Identification and characterization of the feeding circuit-activating peptides, a novel neuropeptide family of Aplysia. J Neurosci 22:7797-7808.

Tian LM, Kawai R, Crow T (2006) Serotonin-immunoreactive CPT interneurons in Hermissenda: identification of sensory input and motor projections. J Neurophysiol 96:327-335.

Vanderwolf CH, Robinson TE (1981) Reticulo-cortical activity and behavior - a critique of the arousal theory and a new synthesis. Behav Brain Sci 4:459-476.

Walters ET, Byrne JH, Carew TJ, Kandel ER (1983) Mechanoafferent neurons innervating tail of Aplysia. II. Modulation by sensitizing stimulation. J Neurophysiol 50:1543-1559.

Weinreich D (1977) Synaptic responses mediated by identified histaminecontaining neurones. Nature 267:854-856.

Weinreich D, McCaman MW, McCaman RE, Vaughn JE (1973) Chemical, enzymatic and ultrastructural characterization of 5-hydroxytryptaminecontaining neurons from the ganglia of Aplysia californica and Tritionia diomedia. J Neurochem 20:969-976.

Weinreich D, Weiner C, McCaman R (1975) Endogenous levels of histamine in single neurons isolated from CNS of Aplysia calfornica. Brain Res 84:341-345.

Weiss KR, Kupfermann I (1976) Homology of the giant serotonergic neurons (metacerebral cells) in Aplysia and pulmonate molluscs. Brain Res 117:33-49.

Weiss KR, Cohen JL, Kupfermann I (1978) Modulatory control of buccal musculature by a serotonergic neuron (metacerebral cell) in Aplysia. J Neurophysiol 41:181-203.

Weiss KR, Mandelbaum DE, Schonberg M, Kupfermann I (1979) Modulation of buccal muscle contractility by serotonergic metacerebral cells in Aplysia: evidence for a role of cyclic adenosine monophosphate. J Neurophysiol 42:791-803.

Weiss KR, Koch UT, Koester J, Rosen SC, Kupfermann I (1982) The role of arousal in modulating feeding behavior of Aplysia: Neural and behavioral studies. In: The neural basis of feeding and reward (Hoebel BG, Novin D, eds), pp 25-57. Brunswick, ME: Haer Institute.

Weiss KR, Shapiro E, Kupfermann I (1986a) Modulatory synaptic actions of an identified histaminergic neuron on the serotonergic metacerebral cell of Aplysia. J Neurosci 6:2393-2402.

Weiss KR, Chiel HJ, Koch U, Kupfermann I (1986b) Activity of an identified histaminergic neuron, and its possible role in arousal of feeding behavior in semi-intact Aplysia. J Neurosci 6:2403-2415.

Weiss KR, Chiel HJ, Kupfermann I (1986c) Sensory function and gating of histaminergic neuron C2 in Aplysia. J Neurosci 6:2416-2426.

Wu JS, Due MR, Sasaki K, Proekt A, Jing J, Weiss KR (2007) State dependence of spike timing and neuronal function in a motor pattern generating network. J Neurosci 27:10818-10831.

Xin Y, Koester J, Jing J, Weiss KR, Kupfermann I (2001) Cerebralabdominal interganglionic coordinating neurons in Aplysia. J Neurophysiol 85:174-186.

Zhang W, Linden DJ (2003) The other side of the engram: experience-driven changes in neuronal intrinsic excitability. Nat Rev Neurosci 4:885-900. 